\title{
Multivalued Lyapunov functions for homeomorphisms of the 2-torus
}

\author{
by \\ Patrice Le Calvez (Paris)
}

\begin{abstract}
Let $F$ be a homeomorphism of $\mathbb{T}^{2}=\mathbb{R}^{2} / \mathbb{Z}^{2}$ isotopic to the identity and $f$ a lift to the universal covering space $\mathbb{R}^{2}$. We suppose that $\kappa \in H^{1}\left(\mathbb{T}^{2}, \mathbb{R}\right)$ is a cohomology class which is positive on the rotation set of $f$. We prove the existence of a smooth Lyapunov function of $f$ whose derivative lifts a non-vanishing smooth closed form on $\mathbb{T}^{2}$ whose cohomology class is $\kappa$.
\end{abstract}

0. Introduction. Let us recall first some classical results mainly due to Schwartzman [Sc] and Fried [Fri]. For convenience we recall these results in the smooth case. Let $\xi$ be a complete smooth (i.e. $C^{\infty}$ ) vector field on an oriented compact connected manifold $M$ and $\left(\phi_{t}\right)_{t \in \mathbb{R}}$ the flow induced by $\xi$. The set of Borel probability measures invariant under the flow is a non-empty convex subset $\mathcal{M}_{\xi}$ of the set of complex measures and is compact with respect to the weak topology. The rotation vector of $\mu \in \mathcal{M}_{\xi}$ may be defined as follows. Take a smooth closed 1-form $\omega$ and write, for $t>0$,

$$
\begin{aligned}
\int_{M}\langle\omega(z), \xi(z)\rangle d \mu(z) & =\frac{1}{t} \int_{0}^{t}\left(\int_{M}\langle\omega(z), \xi(z)\rangle d \phi_{s *} \mu(z)\right) d s \\
& =\frac{1}{t} \int_{M}\left(\int_{\gamma_{z}^{t}} \omega\right) d \mu(z),
\end{aligned}
$$

where $\gamma_{z}^{t}: s \mapsto \phi_{s}(z)$ is defined on $[0, t]$. If $\omega=d H$ is exact the integral may be written

$$
\frac{1}{t} \int_{M}\left(H\left(\phi_{t}(z)\right)-H(z)\right) d \mu(z)=0 .
$$

One deduces that the previous integral depends linearly on the cohomology class $[\omega] \in H^{1}(M, \mathbb{R})$. Thus there exists a homology class $\varrho(\mu) \in H_{1}(M, \mathbb{R})$,

2000 Mathematics Subject Classification: 37B25, 37E30, 37E35, 37E45.

Key words and phrases: homeomorphism of the torus, foliation, rotation vector, rotation set, brick decomposition. 
called the rotation vector of $\mu$, such that

$$
\int_{M}\langle\omega(z), \xi(z)\rangle d \mu(z)=\langle[\omega], \varrho(\mu)\rangle .
$$

The map $\mu \mapsto \varrho(\mu)$ is a continuous affine map and its image $\varrho(\xi)$, called the rotation set of the flow, is a non-empty convex compact subset of $H_{1}(M, \mathbb{R})$.

In the case where $0 \notin \varrho(\xi)$, one can find classes $\kappa \in H^{1}(M, \mathbb{R})$ such that $\langle\kappa, \varrho(\mu)\rangle>0$ for every $\mu \in \mathcal{M}_{\xi}$. Fix such a class $\kappa$, a smooth closed form $\omega$ satisfying $[\omega]=\kappa$, and write $\omega_{t}=t^{-1} \int_{0}^{t} \phi_{s}^{*} \omega d s$ for $t>0$. Then $\left[\omega_{t}\right]=\kappa$ because $\left[\phi_{s}^{*} \omega\right]=\kappa$. Observe that $\left\langle\omega_{t}(z), \xi(z)\right\rangle>0$ for every $z \in M$, if $t$ is large enough. Otherwise, one could find a sequence $\left(t_{n}\right)_{n \geq 0}$ tending to $+\infty$ and a sequence $\left(z_{n}\right)_{n \geq 0}$ in $M$ such that

$$
\left\langle\omega_{t_{n}}\left(z_{n}\right), \xi\left(z_{n}\right)\right\rangle=\int_{\gamma_{z_{n}}^{t_{n}}} \omega \leq 0
$$

Writing $\mu_{n}$ for the equidistributed probability measure defined on the segment of orbit $\gamma_{z_{n}}^{t_{n}}$ and choosing a limit point of the sequence $\left(\mu_{n}\right)_{n \geq 0}$ with respect to the weak topology one would find an invariant measure $\mu$ satisfying $\langle[\omega], \varrho(\mu)\rangle \leq 0$.

Replacing $\omega$ by $\omega_{t}$, one gets:

For any cohomology class $\kappa$ which is positive on $\varrho(\xi)$, one can find a non-vanishing smooth form $\omega$ satisfying $[\omega]=\kappa$ and $\int_{\gamma_{z}^{t}} \omega>0$ for every $z \in M$ and every $t>0$.

In the case where $0 \notin \varrho(\xi)$, one can find a class $\kappa \in H^{1}(M, \mathbb{Z})$ which is positive on $\varrho(\xi)$. The form $\omega$ defined above may be written $\omega=d H$ where $H: M \rightarrow \mathbb{T}^{1}=\mathbb{R} / \mathbb{Z}$ is a smooth submersion: there exists a foliation which is transverse to $\xi$ such that all leaves are diffeomorphic closed hypersurfaces, so the manifold $M$ fibers over $\mathbb{T}^{1}$.

Do similar results exist if one replaces flows by isotopies of the identity?

Suppose that $t \mapsto F_{t}$ is a continuous arc joining $\operatorname{Id}_{M}$ to $F$ in the set of homeomorphisms of $M$ (endowed with the $C^{0}$-topology) and define the trajectory $\gamma_{z}: t \mapsto F_{t}(z)$ of any point $z \in M$. Here again the set $\mathcal{M}_{F}$ of Borel probability measures invariant under $F$ is a non-empty convex and compact set. For any smooth closed 1-form $\omega, \int_{\gamma_{z}} \omega$ may be defined by approximation of $\gamma_{z}$ by a smooth arc. Similarly one defines the rotation vector $\varrho(\mu)$ of any measure $\mu \in \mathcal{M}_{F}$ by

$$
\int_{M}\left(\int_{\gamma_{z}} \omega\right) d \mu(z)=\langle[\omega], \varrho(\mu)\rangle .
$$

The set of rotation vectors of probability invariant measures is a non-empty convex compact subset of $H_{1}(M, \mathbb{R})$. 
In the case where $F$ is smooth, if $\kappa \in H^{1}(M, \mathbb{R})$ satisfies $\langle\kappa, \varrho(\mu)\rangle>0$ for any $\mu \in \mathcal{M}_{F}$ and if $\omega$ is a smooth closed form such that $[\omega]=\kappa$, then $\omega_{n}=n^{-1} \sum_{k=0}^{n-1} F^{k *}(\omega)$ satisfies $\left[\omega_{n}\right]=\kappa$ for any integer $n$ and $\int_{\gamma_{z}} \omega_{n}>0$ for every $z \in M$, if $n$ is large enough. In contrast to the continuous case, one cannot assert that $\omega_{n}$ does not vanish, so it is natural to ask:

Does there exist a non-vanishing smooth 1 -form $\omega$ such that $[\omega]=\kappa$ and $\int_{\gamma_{z}} \omega>0$ for every $z \in M$ ?

and therefore:

Does the existence of a map isotopic to the identity whose rotation set does not contain zero imply that $M$ fibers over $\mathbb{T}^{1}$ ?

We will see that the first question has a positive answer in the case of a surface. If $M$ is a sphere the homology is trivial. If the genus of $M$ is greater than 1 then, by the Lefschetz-Nielsen formula (see Jiang $[\mathrm{J}]$ ), $F$ has a contractible fixed point $z$ (i.e. such that $\gamma_{z}$ is homotopic to zero). The rotation vector of the Dirac measure $\delta_{z}$ is zero. Thus, the second question has a positive answer for surfaces and the only case where the first question has to be considered is the case of the torus $\mathbb{T}^{2}=\mathbb{R}^{2} / \mathbb{Z}^{2}$.

If $F$ is a homeomorphism of $\mathbb{T}^{2}$ which is isotopic to the identity, any isotopy from identity to $F$ can be lifted to the universal covering space $\mathbb{R}^{2}$ to an isotopy of the identity defining a lift $f$ of $F$. The rotation vector of $\mu \in \mathcal{M}_{F}$ may be written

$$
\varrho(\mu)=\int_{\mathbb{T}^{2}} \psi d \mu \in \mathbb{R}^{2} \simeq H_{1}\left(\mathbb{T}^{2}, \mathbb{R}\right),
$$

where $\psi: \mathbb{T}^{2} \rightarrow \mathbb{R}^{2}$ lifts to $f-\operatorname{Id}_{\mathbb{R}^{2}}$ and one defines similarly the rotation set $\varrho(f)$ (there are other ways to define that set, see for example MisiurewiczZiemian $[\mathrm{MZ}])$.

We write $\pi: \mathbb{R}^{2} \rightarrow \mathbb{T}^{2}$ for the universal covering projection. The group of covering transformations is the group $G$ of integer translations $T: z \mapsto z+\mathbf{r}$, $\mathbf{r} \in \mathbb{Z}^{2}$, and may be naturally identified with $H_{1}\left(\mathbb{T}^{2}, \mathbb{Z}\right)$, thus we will write $\langle\kappa, T\rangle$ if $\kappa \in H^{1}\left(\mathbb{T}^{2}, \mathbb{R}\right)$ and $T \in G$.

Let us state the result proved in this paper:

TheOrem 0.1. Let $F$ be a homeomorphism of $\mathbb{T}^{2}$ isotopic to the identity and $f$ a lift of $F$ to $\mathbb{R}^{2}$. Suppose that $\kappa \in H^{1}(M, \mathbb{R})$ is positive on $\varrho(f)$. Then there exists a non-vanishing smooth closed form $\omega$ on $\mathbb{T}^{2}$ satisfying $[\omega]=\kappa$ and such that $H(f(z))-H(z)>0$ for every $z \in \mathbb{R}^{2}$, where $H: \mathbb{R}^{2} \rightarrow \mathbb{R}$ is a primitive of $\pi^{*}(\omega)$.

The theorem above may be stated in a different but equivalent way that we now explain. For that, let us introduce some definitions. 
A line $\Gamma \subset \mathbb{R}^{2}$ is the image of a proper topological embedding of the oriented line $\mathbb{R}$. By the Schoenflies Theorem, the complement $\mathbb{R}^{2} \backslash \Gamma$ has two connected components $R(\Gamma)$ and $L(\Gamma)$, the right and left of $\Gamma$. We will use a partial order $\preceq$ defined on the set of lines, writing $\Gamma \preceq \Gamma^{\prime}$ if $R(\Gamma) \subset R\left(\Gamma^{\prime}\right)$. A Brouwer line of an orientation preserving homeomorphism $f$ of $\mathbb{R}^{2}$ is a line $\Gamma$ such that

$$
f^{-1}(\Gamma) \subset R(\Gamma), \quad f(\Gamma) \subset L(\Gamma),
$$

or equivalently a line $\Gamma$ such that $f(\overline{L(\Gamma)}) \subset L(\Gamma)$ (see [LeC2] for more details on these definitions).

If $\kappa$ is a rational class, Theorem 0.1 asserts that there exists a smooth oriented foliation of $\mathbb{T}^{2}$ with closed leaves which lifts to the plane as a foliation by Brouwer lines all of them invariant under the translations $T \in G$ such that $\langle\kappa, T\rangle=0$. If $\kappa$ is irrational and generated by the form $\alpha d x_{1}-d x_{2}$, Theorem 0.1 asserts that there exists a smooth oriented foliation of $\mathbb{T}^{2}$ smoothly conjugate to a linear foliation which lifts to a foliation of $\mathbb{R}^{2}$ by Brouwer lines with asymptotic irrational slope $\alpha$.

Under the hypothesis of Theorem 0.1, the map $f$ is fixed point free. The Brouwer Plane Translation Theorem asserts that every point belongs to a Brouwer line $[\mathrm{Br}]$. Franks gave a proof of that theorem using Lyapunov functions. More precisely, he proved in [Fra] that an orientation preserving homeomorphism $f$ of $\mathbb{R}^{2}$ which is fixed point free admits a smooth Lyapunov function $H$ which is a Morse function. Moreover, if $z$ is any given point, the level set containing $z$ may be supposed to be a properly embedded line. The important fact in his proof is that there is no chain recurrent point. With the same hypothesis on $f$, we constructed in [LeC2] a topological oriented foliation $\mathcal{F}$ by Brouwer lines and explained in [LeC3] how to make it invariant under $G$ in the case where $f$ commutes with every element of $G$; this was previously known for diffeomorphisms (see [LeC1]). Here the stronger hypothesis on $f$ permits us to obtain stronger dynamical properties on $\mathcal{F}$. It may happen that $f$ is fixed point free but its rotation set contains 0 . It is proved in [LeC1] (but similar results exist in the topological case) that the foliation $\widetilde{\mathcal{F}}$ induced on $\mathbb{T}^{2}$ has both closed and non-closed leaves. If there are Reeb components, there is a finite (and even) number of such components and each Reeb component is an attracting or a repulsing annulus. In that case, there exists $r \in \mathbb{Z}^{2} \backslash\{0\}$ such that every closed leaf joins a point $z$ to $z+r$ or to $z-r$ when lifted to $\mathbb{R}^{2}$. In that case, the rotation set is a segment of the line directed by $r$, and 0 is not an end of it. Moreover the support of any invariant ergodic measure is included in a component of the complement of the union of the closed leaves. If there is no Reeb component, there exists $r \in \mathbb{Z}^{2} \backslash\{0\}$ such that every closed leaf joins a point $z$ to $z+r$ when lifted to $\mathbb{R}^{2}$. The rotation set is included in the half plane to the left of 
the oriented line generated by $r$. In the case where $0 \notin \varrho(f)$, it was proved in [LeC1] and [LeC3] that there exists an equivariant foliation by Brouwer lines; here we prove the existence of many such foliations, the asymptotic direction may be chosen in a well determined open cone.

The method of proof will be similar to but easier than the one in [LeC2] and [LeC3] and will be based on the properties of brick decompositions introduced by Flucher [Fl] and studied in detail by Sauzet [LS], [Sa]. One can find other applications of brick decompositions to the dynamics of surface homeomorphisms in Bonino [Bo], Leroux [LeR] or Béguin, Crovisier, Leroux and Patou [BCLP].

I would like to thank the referee whose numerous remarks have improved the presentation of this article.

1. Brick decompositions. Everything which appears in this section, with the exception of the last part, containing Lemma 1.1, can be found with more details in [Sa], [LeC2] or [LeC3].

Consider a one-dimensional stratified set $\Sigma$ on a (not necessarily closed) surface $M$ with a zero-dimensional submanifold $V$ such that any vertex $v \in V$ is locally the end of exactly three edges. An open edge is a connected component of $\Sigma \backslash V$ and an edge is the closure of an open edge. It is the image of a proper topological embedding of $[0,1],\left[0,+\infty\left[, \mathbb{R}\right.\right.$ or $\mathbb{T}^{1}$. An open brick is a connected component of $M \backslash \Sigma$ and a brick is the closure of an open brick. Writing $E$ (resp. $B$ ) for the set of edges (resp. bricks) one obtains a brick decomposition $\mathcal{D}=(V, E, B)$ of $M$ whose skeleton is $\Sigma=\Sigma(\mathcal{D})$. An example of a brick decomposition is given in Fig. 1.

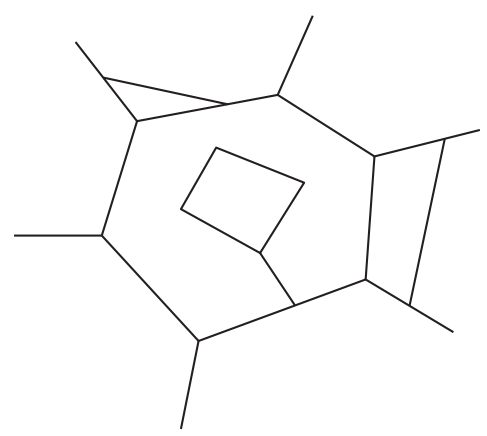

Fig. 1

If $\mathcal{D}^{\prime}=\left(V^{\prime}, E^{\prime}, B^{\prime}\right)$ is a brick decomposition satisfying $\Sigma\left(\mathcal{D}^{\prime}\right) \subset \Sigma(\mathcal{D})$, we say that $\mathcal{D}^{\prime}$ is a subdecomposition of $\mathcal{D}$ and write $\mathcal{D}^{\prime} \subset \mathcal{D}$. Note that one may have $B^{\prime}=B$ even if $\mathcal{D}^{\prime} \neq \mathcal{D}$. 
Suppose that $M$ is a normal covering space of a surface $\widetilde{M}$ and write $\Pi$ : $M \rightarrow \widetilde{M}$ for the projection. If $\widetilde{\mathcal{D}}=(\widetilde{V}, \widetilde{E}, \widetilde{B})$ is a brick decomposition of $\widetilde{M}$, then $\Pi^{-1}(\Sigma(\widetilde{\mathcal{D}}))$ is the skeleton of a brick decomposition $\mathcal{D}=(V, E, B)$ of $M$. We call it the lifted decomposition and write $\mathcal{D}=\Pi^{-1}(\widetilde{\mathcal{D}})$. A brick decomposition $\mathcal{D}=(V, E, B)$ is a lifted decomposition if and only if the sets $V, E$ and $B$ are invariant under the action of the group $G$ of covering transformations, i.e., if $\mathcal{D}$ is a $G$-equivariant decomposition.

Let $\mathcal{D}=(V, E, B)$ be a brick decomposition of $M$. For any $X \subset B$ the union of bricks which are in $X$ is a subsurface of $M$ with boundary. Any connected component of the boundary is contained in $\Sigma(\mathcal{D})$, it is the image of a proper topological embedding of $\mathbb{R}$ or $\mathbb{T}^{1}$. Our subsurface is connected if and only if for any bricks $b, b^{\prime} \in X$, there exists a sequence $\left(b_{i}\right)_{0 \leq i \leq n}$, with $b_{0}=b$ and $b_{n}=b^{\prime}$, such that $b_{i}$ and $b_{i+1}$ are adjacent for $i \in\{0, \ldots, n-1\}$, that is, contain a common edge. For convenience we will denote by the same letter the set $X \subset B$ and the union $X \subset M$ of bricks of $X$, specifying it if necessary if we want to avoid any confusion.

If $\left(X_{i}\right)_{i \in I}$ is a partition of $B$ into connected subsets, the set $\bigcup_{i \in I} \partial X_{i}$ is the skeleton of a subdecomposition $\mathcal{D}^{\prime}$ of $\mathcal{D}$ whose bricks are the $X_{i}$. Let us say that $\mathcal{D}$ is a filled decomposition if $\mathcal{D}^{\prime}=\mathcal{D}$ where $\mathcal{D}^{\prime}$ is defined by the partition into singletons. In other words, $\mathcal{D}$ is filled if and only if any edge is contained in exactly two bricks of the decomposition (for example the brick decomposition in Fig. 1 is not filled).

Suppose that $f$ is a homeomorphism of $M$. Write $\mathcal{P}(B)$ for the set of subsets of $B$. One gets a natural map $\varphi: \mathcal{P}(B) \rightarrow \mathcal{P}(B)$ by defining

$$
\begin{aligned}
\varphi(X) & =\left\{b \in B \mid \text { there exists } b^{\prime} \in X \text { such that } b \cap f\left(b^{\prime}\right) \neq \emptyset\right\} \\
& =\{b \in B \mid b \cap f(X) \neq \emptyset\} .
\end{aligned}
$$

It sends connected subsets to connected subsets and satisfies

$$
\varphi\left(\bigcup_{i \in I} X_{i}\right)=\bigcup_{i \in I} \varphi\left(X_{i}\right), \quad \varphi\left(\bigcap_{i \in I} X_{i}\right) \subset \bigcap_{i \in I} \varphi\left(X_{i}\right),
$$

for any family $\left(X_{i}\right)_{i \in I}$.

Any set $X \subset B$ such that $\varphi(X) \subset X$, or equivalently $f(X) \subset \operatorname{Int}(X)$ in $M$, is called an attractor.

The union or intersection of a family of attractors is again an attractor.

Similarly, one defines $\varphi_{-}: \mathcal{P}(B) \rightarrow \mathcal{P}(B)$ by

$$
\begin{aligned}
\varphi_{-}(X) & =\left\{b \in B \mid \text { there exists } b^{\prime} \in X \text { such that } b \cap f^{-1}\left(b^{\prime}\right) \neq \emptyset\right\} \\
& =\left\{b \in B \mid b \cap f^{-1}(X) \neq \emptyset\right\} .
\end{aligned}
$$

The relations $b^{\prime} \in \varphi^{n}(\{b\})$ and $b \in \varphi_{-}^{n}\left(\left\{b^{\prime}\right\}\right)$ are equivalent. They mean that there exists a sequence $\left(b_{i}\right)_{0 \leq i \leq n}$ of bricks such that $b_{0}=b, b_{n}=b^{\prime}$ and 
$f\left(b_{i}\right) \cap b_{i+1} \neq \emptyset$ for every $i \in\{0, \ldots, n-1\}$. A repulsor is a set positively invariant under $\varphi_{-}$, or equivalently the complement (in $B$ ) of an attractor.

Suppose now that $M=\mathbb{R}^{2}$ is the universal covering space of $\mathbb{T}^{2}$ and that $f$ is the lift of a homeomorphism $F$ of $\mathbb{T}^{2}$ isotopic to the identity. Fix $\kappa \in$ $H^{1}\left(\mathbb{T}^{2}, \mathbb{R}\right)$ and say that a $G$-equivariant brick decomposition $\mathcal{D}=(V, E, B)$ is $\kappa$-positive if:

(i) for all $b \in B, n \geq 1$ and $T \in G$,

$$
T(b) \in \varphi^{n}(\{b\}) \Rightarrow\langle\kappa, T\rangle>0 ;
$$

(ii) for $b \in B$ and $T \in G$,

$$
T(b)=b \Rightarrow\langle\kappa, T\rangle=0 .
$$

Lemma 1.1. If $\kappa \in H^{1}(M, \mathbb{R})$ is positive on $\varrho(f)$, then there exists a $G$-equivariant brick decomposition of $\mathbb{R}^{2}$ which is $\kappa$-positive.

Proof. For any $\varepsilon>0$, one can find a brick decomposition of $\mathbb{T}^{2}$ whose bricks have diameter $\leq \varepsilon$. Let us prove that it lifts to a $\kappa$-positive decomposition if $\varepsilon$ is sufficiently small. Condition (ii) is clearly satisfied for $\varepsilon$ small enough. Suppose that (i) is not. One can find a sequence $\left(\varepsilon_{m}\right)_{m \geq 0}$ converging to zero and for any $m \geq 0$ a brick decomposition $\widetilde{\mathcal{D}}_{m}=\left(\widetilde{V}_{m}, \widetilde{E}_{m}, \widetilde{B}_{m}\right)$ of $\mathbb{T}^{2}$ whose bricks have diameter $\leq \varepsilon_{m}$, a sequence $\left(b_{i}^{m}\right)_{0 \leq i \leq n_{m}}$ of bricks of the lifted decomposition and a translation $T_{m} \in G$ such that:

- $f\left(b_{i}^{m}\right) \cap b_{i+1}^{m} \neq \emptyset$ for any $i \in\left\{0, \ldots, n_{m}-1\right\}$;

- $b_{n_{m}}^{m}=T_{m}\left(b_{0}^{m}\right)$;

- $\left\langle\kappa, T_{m}\right\rangle \leq 0$.

For any $i \in\left\{0, \ldots, n_{m}-1\right\}$ choose $z_{i}^{m} \in b_{i}^{m} \cap f^{-1}\left(b_{i+1}^{m}\right)$, write $\widetilde{z}_{i}^{m}=\pi\left(z_{i}^{m}\right)$ and consider the probability measure

$$
\mu_{m}=\frac{1}{n_{m}} \sum_{i=0}^{n_{m}-1} \delta_{\widetilde{z}_{i}^{m}} .
$$

Because $\varepsilon_{m}$ tends to 0 as $m$ tends to $+\infty$, any limit point $\mu$ of $\left(\mu_{m}\right)_{m \geq 0}$ is invariant under $F$. From $\left\langle\kappa, T_{m}\right\rangle \leq 0$, passing to the limit, one can deduce that the rotation vector (for $f$ ) of $\mu$ satisfies $\langle\kappa, \varrho(\mu)\rangle \leq 0$.

2. The rational case. This is the easiest case. We may suppose that $\kappa$ is represented by the form $d x_{2}$, where $\left(x_{1}, x_{2}\right)$ are the coordinates on the plane. We do not need any brick decomposition. The ideas of this section can be found in $[\mathrm{BCLP}]$. The result stated in the Appendix B of [BCLP], which was previously proved for diffeomorphisms by Kwapisz [Kw], gives a much more precise statement if the rotation set of $f$ is included in $\mathbb{R} \times\left[p / q, p^{\prime} / q^{\prime}\right]$, where $\left.\left[p / q, p^{\prime} / q^{\prime}\right] \subset\right] 0,+\infty[$ is a Farey interval. 
Define $T_{1}: z \mapsto z+(1,0)$ and $T_{2}: z \mapsto z+(0,1)$, denote by $\widehat{f}$ (resp. $\widehat{T}_{2}$ ) the homeomorphism of the annulus $\mathbb{A}=\mathbb{R}^{2} / T_{1}=\mathbb{T}^{1} \times \mathbb{R}$ with lift $f$ (resp. $\left.T_{2}\right)$ and write $\widehat{\kappa}_{1}$ for the generator of $H_{1}(\mathbb{A}, \mathbb{Z})$ associated to $T_{1}$.

By an essential circle we mean a simple oriented closed curve $\Gamma \subset \mathbb{A}$ whose homology class is $\widehat{\kappa}_{1}$. Its complement has two connected components $R(\Gamma)$ and $L(\Gamma)$, the right and left of $\Gamma$. If $\Gamma$ and $\Gamma^{\prime}$ are two essential circles, write

$$
\Gamma \ll \Gamma^{\prime} \quad \text { if } \quad \overline{R(\Gamma)} \subset R\left(\Gamma^{\prime}\right) .
$$

A classical result of Kerékjártó [Ke] asserts that for any pair of essential circles $\Gamma$ and $\Gamma^{\prime}$, there exists an essential circle $\Gamma \wedge \Gamma^{\prime} \subset \Gamma \cup \Gamma^{\prime}$ such that the unbounded connected component of $R(\Gamma) \cap R\left(\Gamma^{\prime}\right)$ is equal to $R\left(\Gamma \wedge \Gamma^{\prime}\right)$ (see [LY] for a modern explanation).

Lemma 2.1. If $\left(\Gamma_{i}\right)_{1 \leq i \leq n}$ and $\left(\Gamma_{i}^{\prime}\right)_{1 \leq i \leq n}$ are two sequences such that $\Gamma_{i} \ll \Gamma_{i}^{\prime}$ for every $i$, then

$$
\bigwedge_{1 \leq i \leq n} \Gamma_{i} \ll \bigwedge_{1 \leq i \leq n} \Gamma_{i}^{\prime}
$$

Proof. The closure of $R\left(\bigwedge_{1<i<n} \Gamma_{i}\right)$ is contained in every $\overline{R\left(\Gamma_{i}\right)}$ and therefore in every $R\left(\Gamma_{i}^{\prime}\right)$. Using the fact that it is connected and unbounded one deduces that it is contained in $R\left(\bigwedge_{1 \leq i \leq n} \Gamma_{i}^{\prime}\right)$.

To get Theorem 0.1 in the rational case one has to prove the following:

Proposition 2.2. There exists a smooth $\widehat{T}_{2}$-invariant foliation of $\mathbb{A}$ by essential circles $\Gamma$ satisfying $\Gamma \ll \widehat{f}(\Gamma)$.

Let us begin with:

Lemma 2.3. There is an integer $n \geq 1$ and a family $\left(\Gamma_{i}\right)_{i \in \mathbb{Z}}$ of essential circles satisfying $\Gamma_{i} \ll \Gamma_{i+1} \ll \widehat{f}\left(\Gamma_{i}\right)$ and $\Gamma_{i+2 n}=\widehat{T}_{2}\left(\Gamma_{i}\right)$ for every $i \in \mathbb{Z}$.

Proof. Write $p_{2}: \mathbb{A} \rightarrow \mathbb{R}$ for the projection on the second factor. Using the fact that $\kappa$ is positive on $\varrho(f)$, one deduces that there exist $\varepsilon>0$ and $N>0$ such that

$$
\frac{\widehat{p}_{2}\left(\widehat{f}^{n}(z)\right)-\widehat{p}_{2}(z)}{n} \geq \varepsilon
$$

for every $n \geq N$ and every $z \in \mathbb{A}$. In particular, one may find $n \geq 1$ such that

$$
\widehat{p}_{2}\left(\widehat{f}^{n}(z)\right)-\widehat{p}_{2}(z)>1 .
$$

One deduces that $\widehat{T}_{2}(\Gamma) \ll \widehat{f}^{n}(\Gamma)$ for any circle $\Gamma=\mathbb{T}^{1} \times\left\{x_{2}\right\}$.

For any $i \in \mathbb{Z}$ write $\Gamma_{i}^{\prime}=\mathbb{T}^{1} \times\{i / 2 n\}$ and define

$$
\Gamma_{i}=\bigwedge_{0 \leq j<n} \widehat{f}^{j}\left(\Gamma_{i-2 j}^{\prime}\right)
$$


Lemma 2.1 shows that $\Gamma_{i} \ll \Gamma_{i+1}$. Clearly, $\Gamma_{i+2 n}=\widehat{T}_{2}\left(\Gamma_{i}\right)$ for every $i \in \mathbb{Z}$. Moreover

$$
\begin{aligned}
& \Gamma_{i+1}=\bigwedge_{0 \leq j<n} \widehat{f}^{j}\left(\Gamma_{i+1-2 j}^{\prime}\right)=\Gamma_{i+1}^{\prime} \wedge \bigwedge_{0 \leq j<n-1} \widehat{f}^{j+1}\left(\Gamma_{i-1-2 j}^{\prime}\right) \\
& \ll \widehat{f}^{n}\left(\Gamma_{i-2 n+1}\right) \wedge \bigwedge_{0 \leq j<n-1} \widehat{f}^{j+1}\left(\Gamma_{i-2 j}^{\prime}\right) \\
& \ll \widehat{f}^{n}\left(\Gamma_{i-2 n+2}\right) \wedge \bigwedge_{0 \leq j<n-1} \widehat{f}^{j+1}\left(\Gamma_{i-2 j}^{\prime}\right) \\
& =\widehat{f}\left(\widehat{f}^{n-1}\left(\Gamma_{i-2 n+2}\right) \wedge \bigwedge_{0 \leq j<n-1} \widehat{f}^{j}\left(\Gamma_{i-2 j}^{\prime}\right)\right)=\widehat{f}\left(\Gamma_{i}\right) .
\end{aligned}
$$

Proof of Proposition 2.2. One can approximate each circle of the family $\left(\Gamma_{i}\right)_{i \in \mathbb{Z}}$ constructed above by a smooth one to get a family of smooth circles satisfying the same property. One can complete this family to a smooth $\widehat{T}_{2}$-invariant foliation of $\mathbb{A}$ by essential circles. For any such circle $\Gamma$, one has necessarily $\Gamma \ll \widehat{f}(\Gamma)$. Indeed, there exists $i \in \mathbb{Z}$ such that

$$
R\left(\Gamma_{i}\right) \subset R(\Gamma) \subset \overline{R(\Gamma)} \subset R\left(\Gamma_{i+1}\right), \quad \overline{R\left(\Gamma_{i+1}\right)} \subset R\left(\widehat{f}\left(\Gamma_{i}\right)\right) \subset R(\widehat{f}(\Gamma)) .
$$

REMark. Consider a homeomorphism of $\mathbb{T}^{2}$ which admits a lift $\widehat{f}$ to A commuting with $\widehat{T}_{2}$, that is, a homeomorphism isotopic to an iterate of the Dehn twist $(x, y) \mapsto(x+y, y)$. For any probability measure $\mu$ invariant under $F$ one may define the vertical rotation number $\varrho_{v}(\mu)=\int_{\mathbb{T}^{2}} \psi d \mu$ where $\psi: \mathbb{T}^{2} \rightarrow \mathbb{R}$ lifts to $\widehat{p}_{2} \circ \widehat{f}-\widehat{f}$. The set of such numbers is a segment, the vertical rotation set $\varrho_{v}(\widehat{f})$ (see Doeff $[\mathrm{D}]$ or Doeff-Misiurewicz [DM] for more details on vertical rotation numbers). Note that the conclusion of Proposition 2.2 is true as soon as $\left.\varrho_{v}(\widehat{f}) \subset\right] 0,+\infty[$.

3. The irrational case, construction of an order. Here again, many arguments of this section can be found with more details in [Sa], [LeC2] or [LeC3].

If $\leq$ is an order on a set $\Theta$ we define, for every $\theta \in \Theta$,

$$
\begin{array}{ll}
(\leftarrow, \theta]_{\leq}=\left\{\theta^{\prime} \in \Theta \mid \theta^{\prime} \leq \theta\right\}, & {[\theta, \rightarrow)_{\leq}=\left\{\theta^{\prime} \in \Theta \mid \theta \leq \theta^{\prime}\right\},} \\
(\leftarrow, \theta)_{\leq}=\left\{\theta^{\prime} \in \Theta \mid \theta^{\prime}<\theta\right\}, & (\theta, \rightarrow)_{\leq}=\left\{\theta^{\prime} \in \Theta \mid \theta<\theta^{\prime}\right\} .
\end{array}
$$

A cut $C=\left(C_{\leftarrow}, C_{\rightarrow}\right)$ is a partition of $\Theta$ into two non-empty subsets such that

$$
\theta \in C_{\leftarrow} \Rightarrow(\leftarrow, \theta]_{\leq} \subset C_{\leftarrow},
$$

or equivalently

$$
\theta \in C_{\rightarrow} \Rightarrow[\theta, \rightarrow)_{\leq} \subset C_{\rightarrow} .
$$


We say that $\theta$ and $\theta^{\prime}$ are comparable if $\theta \leq \theta^{\prime}$ or $\theta^{\prime} \leq \theta$. We say that the order $\leq^{\prime}$ is weaker than $\leq$ if for every $\left(\theta, \theta^{\prime}\right) \in \Theta^{2}$,

$$
\theta \leq \theta^{\prime} \Rightarrow \theta \leq^{\prime} \theta^{\prime}
$$

If $H$ is a group of bijections of $\Theta$, we say that $\leq$ is $H$-equivariant if

$$
\theta \leq \theta^{\prime} \Rightarrow T(\theta) \leq T\left(\theta^{\prime}\right)
$$

for all $\left(\theta, \theta^{\prime}\right) \in \Theta^{2}$ and every $T \in H$.

In the next three sections we suppose that $\kappa \in H^{1}\left(\mathbb{T}^{2}, \mathbb{R}\right)$ is represented by the linear form $\lambda_{\alpha}=-\alpha d x_{1}+d x_{2}$ where $\alpha \notin \mathbb{Q}$. We write

$$
\begin{aligned}
G_{\alpha}^{+} & =\{T \in G \mid\langle\kappa, T\rangle>0\}, \quad G_{\alpha}^{-}=\{T \in G \mid\langle\kappa, T\rangle<0\}, \\
G^{*} & =G \backslash\left\{\operatorname{Id}_{\mathbb{R}^{2}}\right\}=G_{\alpha}^{+} \cup G_{\alpha}^{-} .
\end{aligned}
$$

Let us state the main result of this section:

Proposition 3.1. There exists a G-equivariant brick decomposition $\mathcal{D}=(V, E, B)$ of $\mathbb{R}^{2}$ and a $G$-equivariant order $\leq$ on $B$ which satisfies:

(a) for every $\left(b, b^{\prime}\right) \in B^{2}, f(b) \cap b^{\prime} \neq \emptyset \Rightarrow b<b^{\prime}$;

(b) for every $b \in B$ and $T \in G_{\alpha}^{+}$one has $b<T(b)$;

(c) the order $\leq$ is total;

(d) if $C=\left(C_{\leftarrow}, C_{\rightarrow}\right)$ is a cut of $B$, then both $C_{\leftarrow}$ and $C_{\rightarrow}$ are connected;

(e) for any cut $C$, there exists $M>0$ such that for every $z \in \mathbb{R}^{2}$,

$$
\lambda_{\alpha}(z)<-M \Rightarrow z \in C_{\leftarrow}, \quad \lambda_{\alpha}(z)>M \Rightarrow z \in C_{\rightarrow} .
$$

We will construct $\leq$ step by step. Choose a $\kappa$-positive equivariant brick decomposition $\mathcal{D}=(V, E, B)$ of $\mathbb{R}^{2}$. Recall that:

(i) $T(b) \notin \bigcup_{n>1} \varphi^{n}(\{b\})$ for all $b \in B$ and $T \in G \backslash G_{\alpha}^{+}$;

(ii) $T(b) \neq b$ for all $b \in B$ and $T \in G^{*}$.

Item (ii) is a consequence of the $\kappa$-positivity of $\mathcal{D}$ and of the irrationality of $\kappa$.

We will use very often in this section the fact that, for every $T \in G$, the $\operatorname{map} \varphi: \mathcal{P}(B) \rightarrow \mathcal{P}(B)$ commutes with the map $T: \mathcal{P}(B) \rightarrow \mathcal{P}(B)$ induced by $T$.

Define on $B$ a $G$-equivariant order $\leq_{0}$ in the following way: $b \leq_{0} b^{\prime}$ if $b=b^{\prime}$ or $b<_{0} b^{\prime}$, where

$$
b<_{0} b^{\prime} \Leftrightarrow b^{\prime} \in\left(\bigcup_{n \geq 1} \varphi^{n}(\{b\})\right) \cup\left(\bigcup_{T \in G_{\alpha}^{+}} \bigcup_{n \geq 0} \varphi^{n}(\{T(b)\})\right) .
$$

The relation $\leq_{0}$ is obviously reflexive and transitive. The $\kappa$-positivity of $\mathcal{D}$ implies that $\leq_{0}$ is antisymmetric. Observe that $\leq_{0}$ satisfies the conditions (a) and (b) of Proposition 3.1.

The set of equivariant subdecompositions of $\mathcal{D}$ being finite, one can find a minimal equivariant subdecomposition among the $\kappa$-positive ones. Re- 
placing $\mathcal{D}$ by this minimal decomposition, one may suppose that no strict subdecomposition of $\mathcal{D}$ is $\kappa$-positive. In particular $\mathcal{D}$ is a filled decomposition because the subdecomposition defined by the partition of $B$ into singletons is $\kappa$-positive (it has the same bricks!). Observe also that any brick is bounded by (ii).

LEMmA 3.2. If $b$ and $b^{\prime}$ are two adjacent bricks, then they are comparable.

Proof. This is clear if $b$ and $b^{\prime}$ belong to the same $G$-orbit. If not, write

$$
B^{\prime \prime}=B \backslash \bigcup_{T \in G} T\left(\left\{b, b^{\prime}\right\}\right)
$$

and consider the subdecomposition $\mathcal{D}^{\prime \prime}$ of $\mathcal{D}$ defined by the partition

$$
B=\bigsqcup_{T \in G} T\left(\left\{b, b^{\prime}\right\}\right) \sqcup \bigsqcup_{b^{\prime \prime} \in B^{\prime \prime}}\left\{b^{\prime \prime}\right\} .
$$

It satisfies (ii). We will suppose that $b$ and $b^{\prime}$ are not comparable and prove that (i) is also satisfied by $\mathcal{D}^{\prime \prime}$, which contradicts the minimality of $\mathcal{D}$.

Note that for any sequence $\left(b_{i}\right)_{0 \leq i \leq n}$ in $B$, if we have

- $b_{i+1} \in \varphi\left(\left\{b_{i}\right\}\right)$ for all $i \in\{0, \ldots, n-1\}$;

- $b_{0} \in\left\{b, b^{\prime}\right\}$

- $b_{n} \in\left\{T(b), T\left(b^{\prime}\right)\right\}$;

- $b_{i} \in B^{\prime \prime}$ for all $i \in\{1, \ldots, n-1\}$;

then $T \in G_{\alpha}^{+}$, by $\kappa$-positivity of $\mathcal{D}$ and $b$ and $b^{\prime}$ being incomparable.

By additivity of $G_{\alpha}^{+}$, one deduces that for any sequence $\left(b_{i}\right)_{0 \leq i \leq n}$ in $B$, if we have

- for every $i \in\{0, \ldots, n-1\}$, either

$$
b_{i+1} \in \varphi\left(\left\{b_{i}\right\}\right)
$$

or

$$
b_{i+1} \in \varphi\left(\left\{b_{i}^{\prime}\right\}\right) \text { and }\left\{b_{i}, b_{i}^{\prime}\right\}=T_{i}\left(\left\{b, b^{\prime}\right\}\right) \text { with } T_{i} \in G ;
$$

- $b_{0} \in\left\{b, b^{\prime}\right\}$

- $b_{n} \in\left\{T(b), T\left(b^{\prime}\right)\right\}$;

then $T \in G_{\alpha}^{+}$.

This implies that $\mathcal{D}^{\prime \prime}$ satisfies (i).

Lemma 3.3. For every $b \in B$, there are $b_{1}^{\prime} \in B$ and $b_{2}^{\prime} \in B$ adjacent to $b$ such that $b_{2}^{\prime}<_{0} b<_{0} b_{1}^{\prime}$.

Proof. The set $B_{b}$ of bricks adjacent to $b$ is finite because $b$ is bounded. It contains a maximal element $b_{1}^{\prime} \in B_{b}$ and a minimal element $b_{2}^{\prime}$ for the restricted order. Let us prove that $b<_{0} b_{1}^{\prime}$ (similar arguments give $b_{2}^{\prime}<_{0} b$ ). 
If $b_{1}^{\prime}$ and $b$ belong to the same $G$-orbit, one may write $b_{1}^{\prime}=T(b), T \in G^{*}$, and deduce that $B_{b}$ also contains $T^{-1}(b)$. As $b_{1}^{\prime}=T(b) \nless_{0} T^{-1}(b)$, we deduce that $T \in G_{\alpha}^{+}$and $b<_{0} b_{1}^{\prime}$.

Suppose now that $b_{1}^{\prime}$ and $b$ do not belong to the same $G$-orbit and prove by contradiction that $b<_{0} b_{1}^{\prime}$. Otherwise, by Lemma 3.2, one has $b_{1}^{\prime}<_{0} b$ : there exist $n>0$ and $T \in G \backslash G_{\alpha}^{-}$such that $b \in T\left(\varphi^{n}\left(\left\{b_{1}^{\prime}\right\}\right)\right)$. By maximality of $b_{1}^{\prime}, T\left(\varphi^{n}\left(\left\{b_{1}^{\prime}\right\}\right)\right.$ does not contain any brick adjacent to $b$. As it is connected it reduces to $b$. Thus $T \circ f^{n}\left(b_{1}^{\prime}\right)$ is contained in the interior of $b$, which means that $T \circ f^{n}\left(b^{\prime}\right) \cap b \neq \emptyset$ for any brick $b^{\prime}$ adjacent to $b_{1}^{\prime}$. In particular $T \circ f^{n}(b) \cap b \neq \emptyset$. But $T\left(f^{n}(b)\right) \cap b=\emptyset$ because $\mathcal{D}$ is $\kappa$-positive.

For every brick $b$ choose a point $z_{b} \in \operatorname{Int}(b)$ in such a way that $z_{T(b)}=$ $T\left(z_{b}\right)$ for all $b \in B$ and $T \in G$.

LEMma 3.4. There is a positive number $M_{1}$ such that

$$
b \leq_{0} b^{\prime} \Rightarrow \lambda_{\alpha}\left(z_{b^{\prime}}-z_{b}\right) \geq-M_{1} .
$$

Proof. Write $N$ for the number of bricks of the decomposition of $\mathbb{T}^{2}$ covered by $\mathcal{D}$. Using the equivariance property and a finiteness argument, one can find a positive number $M_{1}$ such that $\lambda_{\alpha}\left(z_{b^{\prime}}-z_{b}\right) \geq-M_{1}$ for all $b \in B$ and $b^{\prime} \in \bigcup_{0 \leq n \leq N-1} \varphi^{n}(\{b\})$. Observe now that

$$
\bigcup_{n \geq 0} \varphi^{n}(\{b\}) \subset \bigcup_{T \notin G_{\alpha}^{-}} T\left(\bigcup_{0 \leq n \leq N-1} \varphi^{n}(\{b\})\right)
$$

and more generally that

$$
[b, \rightarrow)_{\leq_{0}} \subset \bigcup_{T \notin G_{\alpha}^{-}} T\left(\bigcup_{0 \leq n \leq N-1} \varphi^{n}(\{b\})\right) .
$$

Finally, observe that $\lambda_{\alpha}\left(z_{T\left(b^{\prime}\right)}-z_{b^{\prime}}\right) \geq 0$ for all $T \notin G_{\alpha}^{-}$and $b^{\prime} \in B$.

Define on $B$ a $G$-equivariant order $\leq_{1}$ in the following way: $b \leq_{1} b^{\prime}$ if $b=b^{\prime}$ or $b<_{1} b^{\prime}$, where

$$
b<_{1} b^{\prime} \Leftrightarrow \lambda_{\alpha}\left(z_{b^{\prime}}-z_{b}\right) \geq 3 M_{1} .
$$

Lemma 3.5. There is a G-equivariant order $\leq_{2}$ on $B$ which is weaker than $\leq_{0}$ and $\leq_{1}$.

Proof. Write $b \leq_{2} b^{\prime}$ if there exists a sequence $\left(b_{i}\right)_{0 \leq i \leq n}$ such that $b_{0}=b$, $b_{n}=b^{\prime}$ and $b_{i} \leq_{0} b_{i+1}$ or $b_{i} \leq_{1} b_{i+1}$ for $i \in\{0, \ldots, n-1\}$. The relation $\leq_{2}$ is clearly reflexive, transitive and $G$-equivariant. Let us now prove that it is antisymmetric. Suppose that $b \leq_{2} b^{\prime}$ and that the integer $n$ above is smallest possible. Then, for every $i \in\{0, \ldots, n-2\}$, one has $b_{i+1} \leq_{1} b_{i+2}$ if $b_{i} \leq_{0} b_{i+1}$. By Lemma 3.4, this implies that either $b \leq_{0} b^{\prime}$ or $\lambda_{\alpha}\left(z_{b^{\prime}}-z_{b}\right) \geq M_{1}$. This clearly implies that $\leq_{2}$ is antisymmetric. 
Lemma 3.6. If $C=\left(C_{\leftarrow}, C_{\rightarrow}\right)$ is a cut of a $G$-equivariant order $\leq$ on $B$ which is weaker than $\leq_{0}$ and $\leq_{1}$, then $C_{\leftarrow}$ and $C_{\rightarrow}$ are connected. Moreover there exists $M>0$ such that

$$
\lambda_{\alpha}(z) \leq-M \Rightarrow z \in C_{\leftarrow}, \quad \lambda_{\alpha}(z) \geq M \Rightarrow z \in C_{\rightarrow} .
$$

Proof. Fix $b \in C_{\rightarrow}$. The set $[b, \rightarrow)_{\leq 1}$ is included in $C_{\rightarrow}$ because $\leq$ is weaker than $\leq_{1}$. Thus there exists $M>0$ such that $C_{\rightarrow}$ contains $\{z \in$ $\left.\mathbb{R}^{2} \mid \lambda_{\alpha}(z) \geq M\right\}$. This last set, being connected, is included in a connected component $C_{\rightarrow}^{0}$ of $C_{\rightarrow}$. To get the lemma it is sufficient to prove that any connected component $X$ of $C_{\rightarrow}$ meets $C_{\rightarrow}^{0}$. Fix $b^{\prime} \in X$. By Lemma 3.3 one can find a sequence $\left(b_{i}\right)_{i \geq 0}$ starting from $b^{\prime}$ such that $b_{i}<_{0} b_{i+1}$ and $b_{i}$ and $b_{i+1}$ are adjacent. Hence there exists $T \in G$ and two integers $i_{0}<i_{1}$ such that $b_{i_{1}}=T\left(b_{i_{0}}\right)$, and necessarily $T \in G_{\alpha}^{+}$. The order $\leq$being weaker than $\leq_{0}$, each $b_{i}$ belongs to $X$. Moreover $C_{\rightarrow}$ is positively invariant under $T$ because $\leq$ is weaker than $\leq_{0}$ and because $\leq_{0}$ satisfies item (b) of Proposition 3.1. Of course $T$ sends connected components of $C_{\rightarrow}$ to connected components of $C_{\rightarrow}$. Hence $X$ is itself positively invariant under $T$ because $T(X) \cap X \neq \emptyset$. This implies that $X$ meets $\left\{z \in \mathbb{R}^{2} \mid \lambda_{\alpha}(z)>M\right\}$ and therefore meets $C_{\rightarrow}^{0}$. One does the same thing for $C_{\leftarrow}$.

Proof of Proposition 3.1. It is sufficient to find a total $G$-equivariant order $\leq$ on $B$ which is weaker than $\leq_{0}$ and $\leq_{1}$. Using Zorn's Lemma one can find a $G$-equivariant order $\leq$ on $B$ which is weaker than $\leq_{0}$ and $\leq_{1}$ and minimal for this property: there is no strictly weaker $G$-equivariant order than $\leq$. Using the fact that $G$ is an abelian group without torsion which acts freely on $B$, it is not difficult to prove that $\leq$ is a total order. The idea is the following. Suppose that $b^{*}$ and $b^{* *}$ are two bricks which are not comparable. Write $b \mathcal{R}_{1} b^{\prime}$ if there exists a sequence $\left(b_{i}\right)_{0 \leq i \leq n}, n \geq 1$, such that $b_{0}=b, b_{n}=b^{\prime}$ and a sequence $\left(T_{i}\right)_{0 \leq i \leq n}$ in $G$ such that $b_{i} \leq T_{i}\left(b^{*}\right)$ and $T_{i}\left(b^{* *}\right) \leq b_{i+1}$. This relation is equivariant, reflexive, transitive and one has $b^{*} \mathcal{R}_{1} b^{* *}$. One can define $\mathcal{R}_{2}$ in a similar way, replacing $b^{*}$ by $b^{* *}$ and $b^{* *}$ by $b^{*}$. The important fact is that at least one of these two relations is antisymmetric, so it is an equivariant order strictly weaker than $\leq$ (see [LeC3, Section 3] for a detailed proof).

REMARKs. For any brick $b$, we have a partition $B \backslash\{b\}=(\leftarrow, b)_{\leq} \sqcup$ $(b, \rightarrow) \leq$ into connected unbounded subsets. This implies that any brick is a disk.

Observe that if $\leq$ is any order satisfying Proposition 3.1, then for every $b \in B$, there exist $b_{1}^{\prime} \in B$ and $b_{2}^{\prime} \in B$ which are adjacent to $b$ such that $b_{2}^{\prime}<b<b_{1}^{\prime}$. Indeed, both $(\leftarrow, b]_{\leq}$and $[b, \rightarrow)_{\leq}$are connected, unbounded and contain $b$. 
It is the fact that our brick decomposition was minimal among the $\kappa$ positive ones which has permitted us to get Lemmas 3.2 and 3.3 and then Proposition 3.1(d). This last assertion will be fundamental in the construction of a form $\omega$ without singularity in the proof of Theorem 0.1.

The fact that we are working on surfaces has not really been used until now, it will appear in the next sections.

\section{The irrational case, construction of a topological foliation by} Brouwer lines. Let us state the main result proven in this section:

Proposition 4.1. There exists a $G$-equivariant oriented topological foliation of $\mathbb{R}^{2}$ by Brouwer lines with asymptotic slopes equal to $\alpha$.

Let $\mathcal{D}=(V, E, B)$ be a $G$-equivariant brick decomposition on $\mathbb{R}^{2}$ and $\leq$ an order on $B$ satisfying the five conditions of Proposition 3.1. There is a natural orientation of the skeleton $\Sigma(D)$ : every edge $e \in E$ is oriented in such a way that $r(e)<l(e)$, where $r(e)$ (resp. $l(e)$ ) is the brick containing $e$ and located to the right (resp. left) of $e$. Write $v_{-}(e)$ (resp. $\left.v_{+}(e)\right)$ for the initial (resp. final) vertex of the oriented edge $e$.

Let us now define a natural topology on the set $\mathcal{L}_{\mathcal{D}}$ of oriented $\mathcal{D}$ lines, that is, lines $\Gamma \subset \Sigma(D)$ which may be written $\Gamma=\prod_{i \in \mathbb{Z}} e_{i}$, with $v_{+}\left(e_{i}\right)=v_{-}\left(e_{i+1}\right)$. Write $\mathcal{E} \subset E^{\mathbb{Z}}$ for the set of sequences $\left(e_{i}\right)_{i \in \mathbb{Z}}$ such that $v_{+}\left(e_{i}\right)=v_{-}\left(e_{i+1}\right)$ and identify $\mathcal{L}_{\mathcal{D}}$ with the space $\mathcal{E} / \sigma$ of orbits of the shift $\sigma:\left(e_{i}\right)_{i \in \mathbb{Z}} \rightarrow\left(e_{i+1}\right)_{i \in \mathbb{Z}}$. If one endows $E$ with the discrete topology and $E^{\mathbb{Z}}$ with the product topology, one gets a natural (non-Hausdorff) topology on $\mathcal{L}_{\mathcal{D}}$ by taking the quotient topology.

For any cut $C=\left(C_{\leftarrow}, C_{\rightarrow}\right)$, the subsets $C_{\leftarrow} \subset \mathbb{R}^{2}$ and $C_{\rightarrow} \subset \mathbb{R}^{2}$ are connected and unbounded. By Proposition 3.1(d), (e), they have the same boundary which must be a line $\Gamma_{C}$. Since the order $\leq$ satisfies (a), $C_{\rightarrow}$ is an attractor, and we deduce that

$$
f^{-1}\left(\Gamma_{C}\right) \subset \operatorname{Int}\left(C_{\leftarrow}\right), \quad f\left(\Gamma_{C}\right) \subset \operatorname{Int}\left(C_{\rightarrow}\right) .
$$

We get a Brouwer line of $f$ if $\Gamma_{C}$ is oriented in such a way that $R\left(\Gamma_{C}\right)=$ $\operatorname{Int}\left(C_{\leftarrow}\right)$ and $L\left(\Gamma_{C}\right)=\operatorname{Int}\left(C_{\rightarrow}\right)$. This orientation is compatible with the orientation of the skeleton: one may write $\Gamma=\prod_{i \in \mathbb{Z}} e_{i}$ as a concatenation of oriented edges with $v_{+}\left(e_{i}\right)=v_{-}\left(e_{i+1}\right)$. If $C$ and $C^{\prime}$ are two cuts, write $C \preceq C^{\prime}$ if $C_{\leftarrow} \subset C_{\leftarrow}^{\prime}$. The set $\mathcal{C}$ of cuts of $B$ being totally ordered, the set $\mathcal{L}$ of boundary lines $\Gamma_{C}, C \in \mathcal{C}$, is itself totally ordered. Indeed,

$$
C \preceq C^{\prime} \Leftrightarrow R\left(\Gamma_{C}\right) \subset R\left(\Gamma_{C^{\prime}}\right) \Leftrightarrow \Gamma_{C} \preceq \Gamma_{C^{\prime}} .
$$

For every brick $b \in B$, consider the cuts

$$
C_{b}^{-}=\left((\leftarrow, b)_{\leq},[b, \rightarrow)_{\leq}\right), \quad C_{b}^{+}=\left((\leftarrow, b]_{\leq},(b, \rightarrow)_{\leq}\right)
$$


and their respective boundaries $\Gamma_{b}^{-}$and $\Gamma_{b}^{+}$(see Fig. 2). These lines intersect $b$ in two oriented segments $\gamma_{b}^{-}=\prod_{i=0}^{n} e_{i}$ and $\gamma_{b}^{+}=\prod_{i=0}^{n^{\prime}} e_{i}^{\prime}$ with the same initial and final points. We define the initial point of the brick $b$ to be

$$
v_{-}(b)=v_{-}\left(e_{0}\right)=v_{-}\left(e_{0}^{\prime}\right),
$$

and the final point to be

$$
v_{+}(b)=v_{+}\left(e_{n}\right)=v_{+}\left(e_{n}^{\prime}\right) .
$$

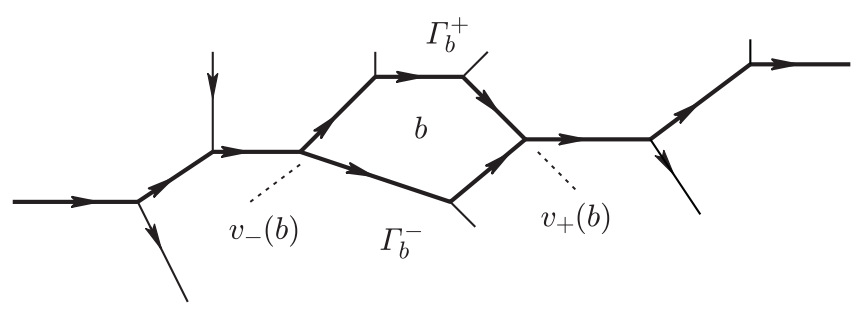

Fig. 2

Proposition 4.2.

(i) The set $\mathcal{L}$ is a closed subset of $\mathcal{L}_{\mathcal{D}}$.

(ii) The restricted topology on $\mathcal{L}$ is the order topology and consequently $\mathcal{L}$ is a Hausdorff space.

(iii) For every edge e the set $\mathcal{L}(e)$ of lines $\Gamma \in \mathcal{L}$ containing e is compact and

$$
\max \mathcal{L}(e)=\Gamma_{l(e)}^{-}, \quad \min \mathcal{L}(e)=\Gamma_{r(e)}^{+} .
$$

(iv) The lines $\Gamma \in \mathcal{L}$ isolated from above (resp. below) are the ones which may be written $\Gamma_{b}^{-}\left(\right.$resp. $\left.\Gamma_{b}^{+}\right)$, where $b \in B$.

Proof. If $\Gamma=\prod_{i \in \mathbb{Z}} e_{i} \in \mathcal{L}_{\mathcal{D}}$ is a limit point of $\mathcal{L}$, there is a sequence $\left(C_{n}\right)_{n \geq 0}$ in $\mathcal{C}$ such that the arc $\prod_{-n \leq i \leq n} e_{i}$ is included in $\Gamma_{C_{n}}$. Writing $C_{n}=\left(C_{\leftarrow}^{n}, C_{\rightarrow}^{n}\right)$, observe that

$$
\limsup \left(C_{n}\right)_{n \geq 0}=\left(\bigcap_{n \geq 0} \bigcup_{k \geq n} C_{\leftarrow}^{n}, \bigcup_{n \geq 0} \bigcap_{k \geq n} C_{\rightarrow}^{n}\right)
$$

is a cut whose boundary is $\Gamma$. This implies that $\mathcal{L}$ is a closed subset of $\mathcal{L}_{\mathcal{D}}$.

The topology of $\mathcal{L}$ is generated by the open sets $\mathcal{L}(e), e \in E$. To prove assertion (ii) observe that for every $e$,

$$
\mathcal{L}(e)=\left\{\Gamma_{C} \mid r(e) \in C_{\leftarrow}, l(e) \in C_{\rightarrow}\right\}=\left\{\Gamma_{C} \mid C_{r(e)}^{-} \prec C \prec C_{l(e)}^{+}\right\},
$$

and that for every cut $C=\left(C_{\leftarrow}, C_{\rightarrow}\right)$,

$$
\left\{\Gamma_{C^{\prime}} \mid C^{\prime} \prec C\right\}=\bigcup_{e \not \subset C \rightarrow} \mathcal{L}(e), \quad\left\{\Gamma_{C^{\prime}} \mid C \prec C^{\prime}\right\}=\bigcup_{e \not \subset C \leftarrow} \mathcal{L}(e) .
$$


The fact that $\mathcal{L}(e)$ is compact is an easy consequence of the following fact: for every edge $e$, there exist at most two edges whose initial points are $v_{+}(e)$ and at most two edges whose final points are $v_{-}(e)$. The second part of assertion (iii) is obvious.

It remains to prove (iv). For any brick $b$, there is no cut strictly contained between $C_{b}^{-}$and $C_{b}^{+}$. The corresponding lines are isolated in $\mathcal{L}$, the first one from above and the second one from below. Suppose that $\Gamma \in \mathcal{L}$ is not the boundary line of a set $C_{b}^{-}$. Write $\Gamma=\prod_{i \in \mathbb{Z}} e_{i}$ and fix $n \geq 0$. Write $b_{n}$ for the smallest brick among the $l\left(e_{i}\right),-n \leq i \leq n$. The boundary line of $C_{b_{n}}^{-}$ is strictly bigger than $\Gamma$ and contains the segment $\prod_{i=-n}^{n} e_{i}$. The sequence $\left(\Gamma_{C_{b_{n}}^{-}}\right)_{n \geq 0}$ converges to $\Gamma$. Similarly if $\Gamma$ is not the boundary line of a set $C_{b}^{+}$, write $b_{n}^{\prime}$ for the largest brick among the $r\left(e_{i}\right),-n \leq i \leq n$, and consider the boundary line of $C_{b_{n}^{\prime}}^{+}$.

Proposition 4.3. One can cover the plane by a totally ordered $G$ equivariant family of Brouwer lines. Any point $z \notin \Sigma(\mathcal{D})$ belongs to a unique such line and the family defines locally near $z$ a topological foliation. Any line of the family passing through $z \in \Sigma(\mathcal{D}) \backslash V$ coincides with the unique edge containing $z$ and here again the family defines locally a topological foliation.

Proof. One can foliate any brick $b$ by a continuous family $\left(\gamma_{b}^{t}\right)_{t \in[-1,1]}$ of segments joining $v_{-}(b)$ to $v_{+}(b)$ which are disjoint except at the ends, and such that $\gamma_{b}^{-1}=\gamma_{b}^{-}$and $\gamma_{b}^{1}=\gamma_{b}^{+}$. One extends each $\gamma_{b}^{t}$ to a line $\Gamma_{b}^{t}$ by adding the two connected components of $\Gamma_{b}^{-} \cap \Gamma_{b}^{+}$. One may suppose that $\gamma_{T(b)}^{t}=T\left(\gamma_{b}^{t}\right)$ for all $b \in B, t \in[-1,1]$ and $T \in G$. The function $t \mapsto \Gamma_{b}^{t}$ is increasing and $\Gamma_{b}^{-1}=\Gamma_{b}^{-}, \Gamma_{b}^{1}=\Gamma_{b}^{+}$. The union of $\mathcal{L}$ and of the set of lines $\Gamma_{b}^{t}, b \in B, t \in(-1,1)$, covers the plane and is totally ordered. Indeed, two lines $\Gamma_{b}^{t}$ and $\Gamma_{b^{\prime}}^{t^{\prime}}$ are clearly comparable if $b=b^{\prime}$. They also are comparable in the case where $b \neq b^{\prime}$. Indeed, if $b<b^{\prime}$, then $\Gamma_{b}^{t} \prec \Gamma_{b}^{+} \preceq \Gamma_{b^{\prime}}^{-} \prec \Gamma_{b^{\prime}}^{t^{\prime}}$. Similarly a line $\Gamma_{b}^{t}$ and a line $\Gamma^{\prime} \in \mathcal{L}$ are comparable. Indeed, either $\Gamma^{\prime} \preceq \Gamma_{b}^{-} \prec \Gamma_{b}^{t}$ or $\Gamma_{b}^{t} \prec \Gamma_{b}^{+} \preceq \Gamma^{\prime}$.

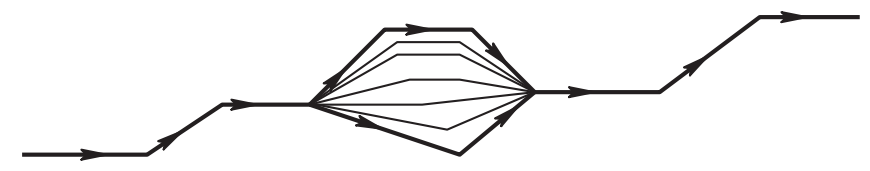

Fig. 3

To get the proposition, one has to prove now that any line $\Gamma_{b}^{t}$ is a Brouwer line. The fact that $\leq$ satisfies condition (a) of Proposition 3.1 implies that $\varphi\left([b, \rightarrow)_{\leq}\right) \subset(b, \rightarrow)_{\leq}$or equivalently that $f\left(\overline{L\left(\Gamma_{b}^{-}\right)}\right) \subset L\left(\Gamma_{b}^{+}\right)$. We deduce 
that for every $t \in[0,1]$ one has

$$
f\left(\overline{L\left(\Gamma_{b}^{t}\right)}\right) \subset f\left(\overline{L\left(\Gamma_{b}^{-}\right)}\right) \subset L\left(\Gamma_{b}^{+}\right) \subset L\left(\Gamma_{b}^{t}\right)
$$

It is possible to desingularize the family given by Proposition 4.3 and to construct a topological oriented foliation on $\mathbb{R}^{2}$ invariant under $G$ such that $\mathcal{L}$ is isomorphic, as an ordered topological space, to a lamination, that is, a closed subset of the set of leaves of our foliation. This foliation will satisfy the conclusion of Proposition 4.1 and project onto a foliation of $\mathbb{T}^{2}$ of Denjoy type. We give here the idea of this desingularization; the details can be found in [LeC2]. One will add bricks close to the skeleton of $\mathcal{D}$.

Say that an edge $e$ is singular if $\mathcal{L}(e)$ reduces to a single line, and regular otherwise. A vertex $v$ cannot be the final point, or the initial point, of three edges (because $\leq$ is an order). There are two types of vertices: a vertex of the first type is the initial point of two edges and the final point of one edge, it is the initial point of a brick; a vertex of the second type is the initial point of one edge and the final point of two edges, it is the final point of a brick.

Fix a vertex $v$ of the first type, and consider the edges $\left(e_{i}\right)_{1 \leq i \leq 3}$ such that

$$
v=v_{+}\left(e_{1}\right)=v_{-}\left(e_{2}\right)=v_{-}\left(e_{3}\right), \quad r\left(e_{3}\right)=l\left(e_{2}\right),
$$

and the brick $b$ such that $v=v_{-}(b)$. Conjugating $f$ by an orientation preserving homeomorphism, one may suppose that close to $v$, the skeleton $\Sigma(\mathcal{D})$ is as in Fig. 4.

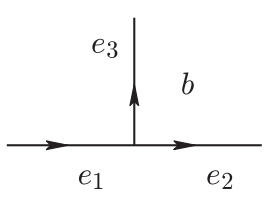

Fig. 4

The edge $e_{1}$ is necessarily regular, it lies on at least two lines of $\mathcal{L}$, one containing $e_{2}$ and the other containing $e_{3}$. If both $e_{2}$ and $e_{3}$ are regular we add the three bricks $\beta_{e_{1}}^{e_{2}}, \beta_{e_{1}}^{b}, \beta_{e_{1}}^{e_{3}}$ drawn in Fig. 5, and denote by $I_{+}\left(e_{1}\right), I_{-}\left(e_{2}\right), I_{-}(b), I_{-}\left(e_{3}\right)$ the four oriented segments as in the figure. Then we foliate the three bricks as indicated and denote by $h_{v}: I_{+}\left(e_{1}\right) \rightarrow$ $I_{-}\left(e_{2}\right) I_{-}(b) I_{-}\left(e_{3}\right)$ the induced homeomorphism. One can construct on each segment $I_{-}\left(e_{i}\right), i \in\{2,3\}$, a closed set $\mathcal{L}_{-}\left(e_{i}\right)$ containing the ends which is isomorphic as an ordered topological space to $\mathcal{L}\left(e_{i}\right)$. Observe that the set defined by $\mathcal{L}_{+}\left(e_{1}\right)=h_{v}^{-1}\left(\mathcal{L}_{-}\left(e_{2}\right) \cup \mathcal{L}_{-}\left(e_{3}\right)\right)$ is isomorphic to $\mathcal{L}\left(e_{1}\right)$.

If $e_{2}$ is regular and $e_{3}$ is singular we add only the two bricks $\beta_{e_{1}}^{e_{2}}, \beta_{e_{1}}^{b}$ introduced above and define in the same way $I_{-}(b), I_{-}\left(e_{2}\right)$ and a shorter 


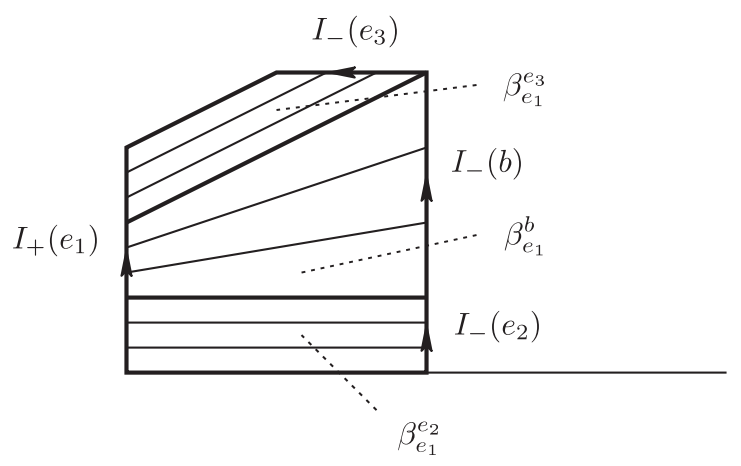

Fig. 5

segment $I_{+}\left(e_{1}\right)$ (see Fig. 6). We foliate the two bricks in the same way and define $h_{v}: I_{+}\left(e_{1}\right) \rightarrow I_{-}\left(e_{2}\right) I_{-}(b)$. The set $\mathcal{L}_{-}\left(e_{3}\right)$ reduces to the final point of $I_{-}(b)$. Construct on $I_{-}\left(e_{2}\right)$ a closed set $\mathcal{L}_{-}\left(e_{2}\right)$ containing the ends which is isomorphic to $\mathcal{L}\left(e_{2}\right)$ and set $\mathcal{L}_{+}\left(e_{1}\right)=h_{v}^{-1}\left(\mathcal{L}_{-}\left(e_{2}\right) \cup \mathcal{L}_{-}\left(e_{3}\right)\right)$.

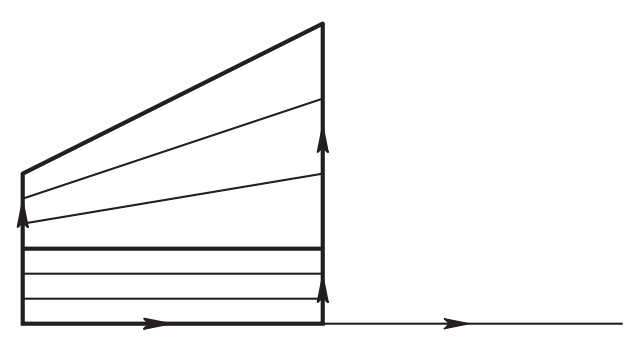

Fig. 6

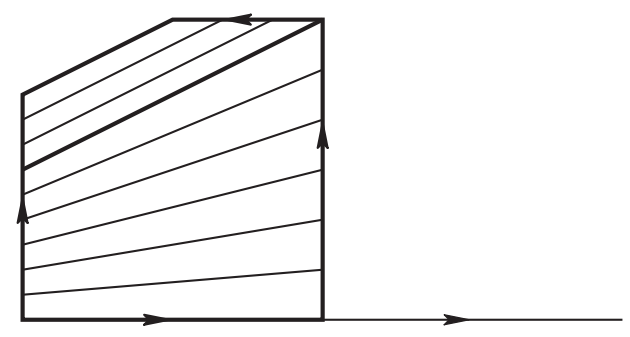

Fig. 7

If $e_{2}$ is singular and $e_{3}$ is regular we add two bricks $\beta_{e_{1}}^{b}, \beta_{e_{1}}^{e_{3}}$ (see Fig. 7), the brick $\beta_{e_{1}}^{b}$ being the union of the bricks $\beta_{e_{1}}^{e_{2}}$ and $\beta_{e_{1}}^{b}$ defined in the first case and the brick $\beta_{e_{1}}^{e_{3}}$ being unchanged. The segments $I_{+}\left(e_{1}\right)$ and $I_{-}\left(e_{3}\right)$ are the same as in the first case and the new segment $I_{-}(b)$ is the union of the previous ones $I_{-}\left(e_{2}\right)$ and $I_{-}(b)$. Foliate the two bricks as in the figure 
and define similarly $h_{v}: I_{+}\left(e_{1}\right) \rightarrow I_{-}(b) I_{-}\left(e_{3}\right)$. The set $\mathcal{L}_{-}\left(e_{2}\right)$ reduces to the initial point of $I_{-}(b)$, the set $\mathcal{L}_{-}\left(e_{3}\right)$ is a closed subset of $I_{-}\left(e_{3}\right)$ containing the ends which is isomorphic to $\mathcal{L}\left(e_{3}\right)$, and we set $\mathcal{L}_{+}\left(e_{1}\right)=$ $h_{v}^{-1}\left(\mathcal{L}_{-}\left(e_{2}\right) \cup \mathcal{L}_{-}\left(e_{3}\right)\right)$.

If $e_{2}$ and $e_{3}$ are both singular add only the brick $\beta_{e_{1}}^{b}$ defined in the previous case (see Fig. 8). The segment $I_{-}(b)$ is unchanged and $I_{+}\left(e_{1}\right)$ is defined as in the second case. Foliate the brick as previously and define $h_{v}: I_{+}\left(e_{1}\right) \rightarrow I_{-}(b)$. Each set $\mathcal{L}_{-}\left(e_{2}\right)$ and $\mathcal{L}_{-}\left(e_{3}\right)$ is an end of $I_{-}(b)$ and $\mathcal{L}_{+}\left(e_{1}\right)=h_{v}^{-1}\left(\mathcal{L}_{-}\left(e_{2}\right) \cup \mathcal{L}_{-}\left(e_{3}\right)\right)$ is the union of the two ends of $I_{+}\left(e_{1}\right)$.

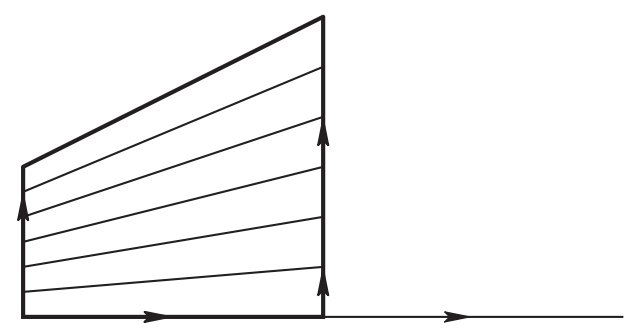

Fig. 8

Consider now a vertex $v$ of the second type, the edges $\left(e_{i}\right)_{1 \leq i \leq 3}$ such that

$$
v=v_{-}\left(e_{1}\right)=v_{+}\left(e_{2}\right)=v_{+}\left(e_{3}\right), \quad r\left(e_{3}\right)=l\left(e_{2}\right),
$$

and the brick $b$ such that $v=v_{+}(b)$. We have a symmetric situation. Let us explain the construction in the case where $e_{2}$ and $e_{3}$ are regular. We construct three foliated bricks $\beta_{e_{2}}^{e_{1}}, \beta_{b}^{e_{1}}, \beta_{e_{3}}^{e_{1}}$, four oriented segments $I_{-}\left(e_{1}\right), I_{+}\left(e_{2}\right), I_{+}(b), I_{+}\left(e_{3}\right)$, and a homeomorphism $h_{v}: I_{+}\left(e_{2}\right) I_{+}(b) I_{+}\left(e_{3}\right)$ $\rightarrow I_{-}\left(e_{1}\right)$. On each segment $I_{+}\left(e_{i}\right), i \in\{2,3\}$, we get a closed set $\mathcal{L}_{+}\left(e_{i}\right)$ containing the ends which is isomorphic to $\mathcal{L}\left(e_{i}\right)$ and finally the set $\mathcal{L}_{-}\left(e_{i}\right)=h_{v}\left(\mathcal{L}_{+}\left(e_{2}\right) \cup \mathcal{L}_{+}\left(e_{3}\right)\right)$ which is isomorphic to $\mathcal{L}\left(e_{1}\right)$ (see Fig. 9).
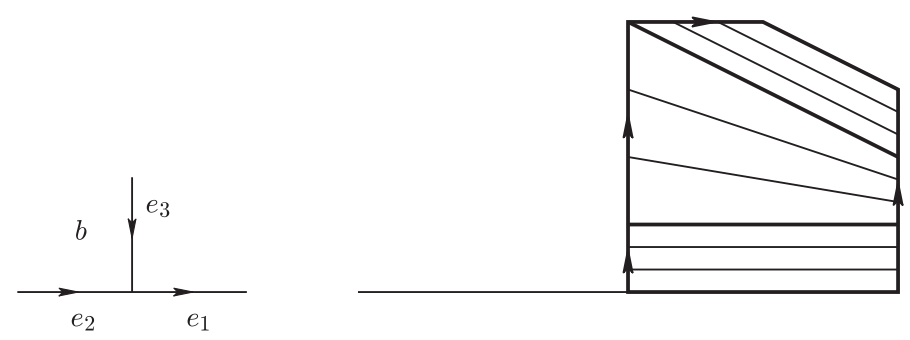

Fig. 9

Consider now a regular edge $e$. One constructs a new brick $\beta_{e} \subset l(e)$ whose boundary contains $I_{-}(e), I_{+}(e)$ and the subarc of $e$ joining the two 
initial points of $I_{-}(e), I_{+}(e)$. We foliate it in such a way that the induced map $h_{e}: I_{-}(e) \rightarrow I_{+}(e)$ sends homeomorphically $\mathcal{L}_{-}(e)$ onto $\mathcal{L}_{+}(e)$. An example is depicted in Fig. 10 after conjugation by an orientation preserving homeomorphism.

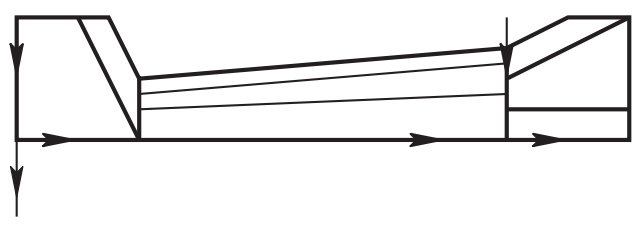

Fig. 10

Finally, consider a brick $b$. Observe that the new bricks which are included in $b$ are the bricks $\beta_{e}$, where $l(e)=b$, and the bricks constructed at a vertex $v$ such that there exist $e$ and $e^{\prime}$ satisfying $v_{+}(e)=v_{-}\left(e^{\prime}\right)=v$ and $l(e)=l\left(e^{\prime}\right)=b$. The closure of the complement in $b$ of the union of these new bricks is a closed disk $\beta_{b}$ whose boundary contains the two segments $I_{-}(b)$ and $I_{+}(b)$. One can foliate the brick $\beta_{b}$ as indicated in Fig. 11 and construct a homeomorphism $h_{b}: I_{-}(b) \rightarrow I_{+}(b)$.

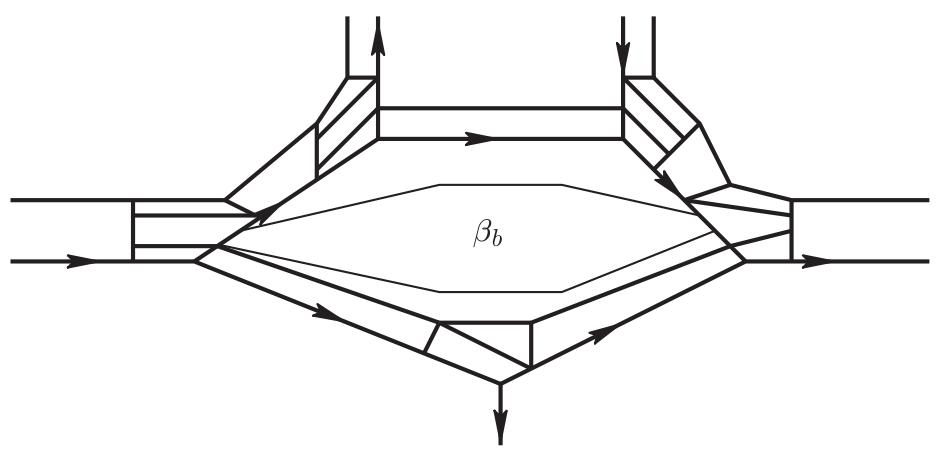

Fig. 11

All the objects defined above may be chosen $G$-equivariant. We have constructed a new equivariant brick decomposition and an oriented topological foliation $\mathcal{F}$ of the plane which lifts an oriented topological foliation $\widetilde{\mathcal{F}}$ of $\mathbb{T}^{2}$. Let us study these foliations. Write $\phi_{z}$ for the leaf passing through a given point $z$.

Recall that if $e$ is an edge, then the ordered topological spaces $\mathcal{L}_{-}(e)$ and $\mathcal{L}_{-}(e)$ are both isomorphic to $\mathcal{L}(e)$. Write $\Psi_{-}(e): \mathcal{L}(e) \rightarrow \mathcal{L}_{-}(e)$ and $\Psi_{+}(e): \mathcal{L}(e) \rightarrow \mathcal{L}_{+}(e)$ for these isomorphisms. If $\Gamma=\prod_{i \in \mathbb{Z}} e_{i}$ is a line in $\mathcal{L}$, the points $\Psi_{-}\left(e_{i}\right)(\Gamma)$ and $\Psi_{+}\left(e_{i}\right)(\Gamma)$ belong to the same leaf of $\mathcal{F}$ and this leaf does not depend on $i$; denote it $\Psi(\Gamma)$. Observe that $\Gamma \preceq \Psi(\Gamma)$. More 
precisely,

$$
R(\Gamma) \subset R(\Psi(\Gamma)) \subset \operatorname{Int}\left(R(\Gamma) \cup \bigcup_{i \in \mathbb{Z}} l\left(e_{i}\right)\right) .
$$

This implies in particular that $\Psi(\Gamma)$ has an asymptotic irrational slope $\alpha$. This also implies that $\Psi(\Gamma) \prec \Psi\left(\Gamma^{\prime}\right)$ if $\Gamma \prec \Gamma^{\prime}$. Indeed, the inequality is obviously true if $\Gamma^{\prime}$ contains an edge $e_{i}$ because $\Psi_{-}\left(e_{i}\right)(\Gamma) \prec \Psi_{-}\left(e_{i}\right)\left(\Gamma^{\prime}\right)$, but it is also true if $\Gamma \cap \Gamma^{\prime}=\emptyset$ because $\operatorname{Int}\left(R(\Gamma) \cup \bigcup_{i \in \mathbb{Z}} l\left(e_{i}\right)\right) \subset R\left(\Gamma^{\prime}\right)$ in that case.

Observe that for any brick $b$, the initial points of $I_{-}(b)$ and $I_{+}(b)$ belong to $\Psi\left(\Gamma^{-}(b)\right)$ and the final points belong to $\Psi\left(\Gamma^{+}(b)\right)$. In particular any leaf $\phi$ which meets the interior of $\beta_{b}$ satisfies $\Psi\left(\Gamma_{-}(b)\right) \prec \phi \prec \Psi\left(\Gamma_{+}(b)\right)$. Recall that if one writes $\Gamma_{b}^{-}=\prod_{i \in \mathbb{Z}} e_{i}^{-}$and $\Gamma_{b}^{+}=\prod_{i \in \mathbb{Z}} e_{i}^{+}$with $v_{-}\left(e_{0}^{-}\right)=v_{-}\left(e_{0}^{+}\right)=$ $v_{-}(b)$, then $e_{i}^{-}=e_{i}^{+}$for every $i<0$. Similarly if one writes $\Gamma_{b}^{-}=\prod_{i \in \mathbb{Z}} e_{i}^{\prime-}$ and $\Gamma_{b}^{+}=\prod_{i \in \mathbb{Z}} e_{i}^{\prime+}$ with $v_{+}\left(e_{0}^{\prime-}\right)=v_{+}\left(e_{0}^{\prime+}\right)=v_{+}(b)$, then $e_{i}^{\prime-}=e_{i}^{\prime+}$ for every $i>0$. This implies that $\phi$ does not meet any other brick $\beta_{b^{\prime}}, b^{\prime} \in B$, and intersects $\beta_{b}$ in a unique segment joining $I_{-}(b)$ to $I_{+}(b)$. In particular $\phi$ projects onto a wandering leaf of $\widetilde{\mathcal{F}}$.

Take a point $z$ in a segment $I_{-}(e)$ which does not belong to $\mathcal{L}_{-}(e)$. Let $\left(z^{-}, z^{+}\right)$be the connected component of $I_{-}(e) \backslash \mathcal{L}_{-}(e)$ containing $z$. The point $z^{-}$(resp. $z^{+}$) is the image under $\Psi$ of a line isolated from above (resp. from below), more precisely there is a brick $b \in B$ such that $z^{-}=\Psi_{-}(e)\left(\Gamma_{b}^{-}\right)$ and $z^{+}=\Psi_{-}(e)\left(\Gamma_{b}^{+}\right)$. We deduce that the leaf $\phi_{z}$ meets the interior of $\beta_{b}$.

In conclusion, there are two kinds of leaves, those which are in the image of $\Psi$ and those which intersect the interior of a brick $\beta_{b}$. The arguments at the beginning of the proof of Proposition 4.3 tell us that the set of leaves is totally ordered. Therefore the foliation is trivial. The set of leaves of the first kind is closed, it is a lamination, isomorphic to $\mathcal{L}$, which projects to a lamination of $\widetilde{\mathcal{F}}$. The foliation is an irrational foliation of Denjoy type with an asymptotic slope $\alpha$.

To get Proposition 4.1 one has to prove:

LEMMA 4.4. If the new bricks are chosen very close to the skeleton, then the leaves of $\mathcal{F}$ are Brouwer lines.

Proof. Remember that for any brick $b$, we have the following inclusions in the plane:

$$
f\left(\overline{L\left(\Gamma_{b}^{-}\right)}\right) \subset L\left(\Gamma_{b}^{+}\right), \quad f^{-1}\left(\overline{R\left(\Gamma_{b}^{+}\right)}\right) \subset R\left(\Gamma_{b}^{-}\right) .
$$

Therefore we can choose $G$-equivariantly a neighborhood $U_{b}$ of $b$ such that

$$
f\left(U_{b}\right) \subset L\left(\Gamma_{b}^{+}\right), \quad f^{-1}\left(U_{b}\right) \subset R\left(\Gamma_{b}^{-}\right) .
$$

Suppose now that the bricks constructed in a neighborhood of a vertex $v$ are included in the three sets $U_{b}$ such that $v \in b$ and that the brick $\beta_{e}$ 
constructed in a neighborhood of a regular edge $e$ is included in the two sets $U_{b}$ such that $e \subset b$. We will prove that any leaf of $\mathcal{F}$ is a Brouwer line of $f$.

For any leaf $\phi=\Psi(\Gamma)$ where $\Gamma=\Gamma_{C}=\prod_{i \in \mathbb{Z}} e_{i}$, we have

$$
R(\Gamma) \subset R(\phi) \subset \overline{R(\phi)} \subset R(\Gamma) \cup \bigcup_{i \in \mathbb{Z}} U_{r\left(e_{i}\right)} .
$$

We know that $R\left(\Gamma_{r\left(e_{i}\right)}^{-}\right) \subset R(\Gamma)$ because $r\left(e_{i}\right) \in C_{\leftarrow}$. We deduce that

$$
\begin{aligned}
f^{-1}(\overline{R(\phi)}) & \subset f^{-1}(R(\Gamma)) \cup \bigcup_{i \in \mathbb{Z}} f^{-1}\left(U_{r\left(e_{i}\right)}\right) \\
& \subset R(\Gamma) \cup \bigcup_{i \in \mathbb{Z}} R\left(\Gamma_{r\left(e_{i}\right)}^{-}\right)=R(\Gamma) \subset R(\phi) .
\end{aligned}
$$

Therefore $\phi$ is a Brouwer line.

Now consider a leaf $\phi$ intersecting the interior of a brick $\beta_{b}$. Write $\Gamma_{b}^{+}=$ $\prod_{i \in \mathbb{Z}} e_{i}$. Observe that

$$
R\left(\Gamma_{b}^{-}\right) \subset R(\phi) \subset \overline{R(\phi)} \subset R\left(\Gamma_{b}^{-}\right) \cup \bigcup_{i \in \mathbb{Z}} U_{r\left(e_{i}\right)} .
$$

Observe that $r\left(e_{i}\right) \leq b$ and therefore $R\left(\Gamma_{r\left(e_{i}\right)}^{-}\right) \subset R\left(\Gamma_{b}^{-}\right)$for every $i \in \mathbb{Z}$. We deduce that

$$
\begin{aligned}
f^{-1}(\overline{R(\phi)}) & \subset f^{-1}\left(R\left(\Gamma_{b}^{-}\right)\right) \cup \bigcup_{i \in \mathbb{Z}} f^{-1}\left(U_{r\left(e_{i}\right)}\right) \\
& \left.\subset R\left(\Gamma_{b}^{-}\right) \cup \bigcup_{i \in \mathbb{Z}} R\left(\Gamma_{r\left(e_{i}\right)}^{-}\right)\right)=R\left(\Gamma_{b}^{-}\right) \subset R(\phi) .
\end{aligned}
$$

Therefore $\phi$ is a Brouwer line.

5. The irrational case, construction of a smooth foliation by Brouwer lines. We now perturb $\mathcal{F}$ to construct a foliation smoothly equivariantly conjugate to the linear foliation of slope $\alpha$. The arguments are rather standard.

Lemma 5.1. There exists a $G$-equivariant brick decomposition $\mathcal{D}^{\prime}=$ $\left(V^{\prime}, E^{\prime}, B^{\prime}\right)$ of $\mathbb{R}^{2}$ and a $G$-equivariant order $\leq$ on $B$ which satisfy items (a)-(e) of Proposition 3.1 and such that:

- any edge e $\in E^{\prime}$ is smooth;

- the model drawn in Fig. 5 shows the structure of $\Sigma\left(\mathcal{D}^{\prime}\right)$ in a neighborhood of a vertex in an affine chart.

Proof. Note first that if we slightly perturb the skeleton of $\mathcal{D}$ and get a new $G$-equivariant decomposition $\mathcal{D}^{\prime}=\left(V^{\prime}, E^{\prime}, B^{\prime}\right)$ with a natural bijection $\delta: B \rightarrow B^{\prime}$, then the order $\delta_{*}(\leq)$ defined on $B^{\prime}$ still has the properties stated in Proposition 3.1. Indeed, the only non-trivial property to verify is (a). But 
we are dealing with closed bricks, therefore if $\mathcal{D}^{\prime}$ is sufficiently "close" to $\mathcal{D}$, then for any bricks $b_{0}, b_{1}$ in $B$,

$$
f\left(b_{0}\right) \cap b_{1}=\emptyset \Rightarrow f\left(\delta\left(b_{0}\right)\right) \cap \delta\left(b_{1}\right)=\emptyset .
$$

The decomposition $\mathcal{D}$ has been constructed as a subdecomposition of any given decomposition $\mathcal{D}^{\prime \prime}$ with very small bricks. One may suppose that every edge of $\mathcal{D}^{\prime \prime}$ is affine and therefore that every edge of $\mathcal{D}$ is piecewise affine. We begin by modifying $\Sigma(\mathcal{D})$ in a neighborhood of any vertex as in Fig. 12, and then we smooth any new edge.
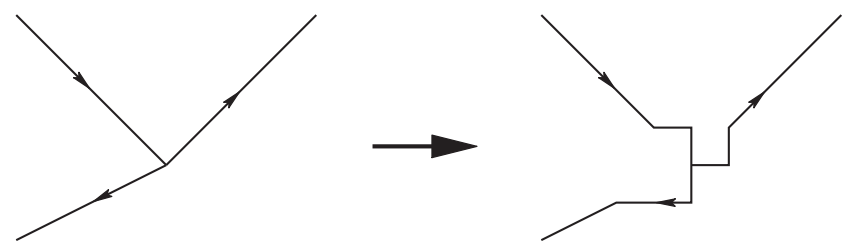

Fig. 12

Lemma 5.2. Our foliation $\mathcal{F}$ may be constructed such that every leaf is smooth and tangent to a certain non-vanishing $G$-equivariant continuous vector field $\xi_{0}$.

Proof. In the desingularization procedure of Section 4, instead of adding affine bricks in a neighborhood of a vertex, we can choose the model of foliated bricks described in Fig. 13.

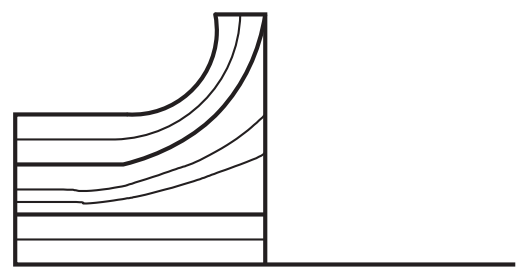

Fig. 13

Fig. 13 is a smoothed version of Fig. 5. Other figures in Section 4 can be smoothed in an analogous way. We can do a similar thing to construct the (non-foliated) bricks $\beta_{e}$ and $\beta_{b}$. There will not be any problem to foliate smoothly the bricks $\beta_{b}$ but there will be for the bricks $\beta_{e}$ (we cannot choose our foliation $\mathcal{F}$ smooth and even $C^{2}$ because of the Denjoy counterexample). Indeed, the maps $h_{v}$ and $h_{b}$ may be chosen smooth but not the map $h_{e}$ because it must send $\mathcal{L}_{-}(e)$ onto $\mathcal{L}_{+}(e)$. Let us see how to foliate $\beta_{e}$ to get the lemma. One should be able to do the following. An orientation 
preserving homeomorphism $h:[0,1] \rightarrow[0,1]$ being given, one has to find a non-vanishing continuous vector field $\xi$ on the plane equal to $(1,0)$ outside $[-1,1] \times[0,1]$ and a continuous oriented foliation with smooth leaves tangent to $\xi$ and such that any leaf passing through $(-1, u)$ contains $(1, h(u))$.

Extend $h$ to $\mathbb{R}$ by setting $h(u)=u$ if $u \notin[0,1]$ and define $\psi_{0}: \mathbb{R}^{2} \rightarrow \mathbb{R}$ by

$$
\psi_{0}(t, u)= \begin{cases}u & \text { if } t \leq-1 / 2, \\ (1 / 2-t) u+(1 / 2+t) h(u) & \text { if } t \in[-1 / 2,1 / 2], \\ h(u) & \text { if } t \geq 1 / 2 .\end{cases}
$$

Then define

$$
\psi_{1}:(t, u) \mapsto \int_{\mathbb{R}} \chi(t-s) \psi_{0}(s, u) d s
$$

where $\chi$ is a smooth non-negative function with support in $[-1 / 2,1 / 2]$ satisfying $\int_{\mathbb{R}} \chi(s) d s=1$, and consider the family of graphs of the partial maps $t \mapsto \psi_{1}(t, u)$.

Observe that the tangent vector field will be $C^{0}$-close to the constant horizontal vector field $(0,1)$ if $h$ is $C^{0}$-close to the identity.

Write $\widetilde{\xi}_{0}$ for the vector field naturally defined on $\mathbb{T}^{2}$ and choose a smooth non-vanishing vector field $\widetilde{\eta}$ satisfying $\widetilde{\xi}_{0}(z) \wedge \widetilde{\eta}(z)>0$, where $\wedge$ is the usual symplectic form. Perturbing $\widetilde{\eta}$ if necessary and rescaling it, one may suppose that the induced flow has a periodic orbit $\widetilde{\Gamma}$ of period 1 . It is a smooth global Poincaré section of $\widetilde{\xi_{0}}$. The preimage of $\widetilde{\Gamma}$ in $\mathbb{R}^{2}$ defines an ordered family $\left(\Gamma_{i}\right)_{i \in \mathbb{Z}}$ of global sections of $\mathcal{F}$, where $\Gamma_{i+1} \prec \Gamma_{i}$. Consider the time parametrization $\tau: \Gamma_{0} \rightarrow \mathbb{R}$ and the translation $T \in G^{*}$ such that $\tau(T(z))=\tau(z)+1$. Choose a translation $T^{\prime} \in G^{*}$ such that $\Gamma_{1}=T^{\prime}\left(\Gamma_{0}\right)$. We clearly have:

Lemma 5.3. There is a neighborhood $\Xi$ of $\xi_{0}$ (for the $C^{0}$-topology) in the set of continuous equivariant vector fields such that:

- any vector field $\xi \in \Xi$ is non-singular;

- if $\xi$ is smooth, the circle $\widetilde{\Gamma}$ is a global section of the foliation of $\mathbb{T}^{2}$ associated to the induced vector field $\widetilde{\xi}$.

For any smooth vector field $\xi \in \Xi$ and for $\xi=\xi_{0}$ we define:

- the foliation $\mathcal{F}_{\xi}$ defined by $\xi$

- the holonomy map $g_{\xi}^{\prime}: \Gamma_{0} \rightarrow \Gamma_{1}$;

- the homeomorphism $g_{\xi}=T^{\prime-1} \circ g_{\xi}^{\prime}$ and its (real) rotation number $\varrho_{\xi} \in \mathbb{R}$ (to be more precise, the rotation number of $\tau \circ g_{\xi} \circ \tau^{-1}$, which is a lift of a homeomorphism of $\mathbb{T}^{1}$ );

- the projection $p_{\xi}: \mathbb{R}^{2} \rightarrow \Gamma_{0}$ which associates to any point $z \in \mathbb{R}^{2}$ the intersection of the leaf $\phi_{z}$ of $\mathcal{F}_{\xi}$ and $\Gamma_{0}$. 
Lemma 5.4. There exists a convex neighborhood $\Xi^{\prime} \subset \Xi$ of $\xi_{0}$ for the $C^{0}$-topology such that for every smooth vector field $\xi \in \Xi^{\prime}$, the leaves of $\mathcal{F}_{\xi}$ are Brouwer lines. More precisely, for every $z \in \mathbb{R}^{2}$,

$$
\tau\left(p_{\xi}(f(z))\right)-\tau\left(p_{\xi}(z)\right)>0 .
$$

Proof. The leaves of $\mathcal{F}=\mathcal{F}_{\xi_{0}}$ being Brouwer lines, for any $z \in \mathbb{R}^{2}$ one has

$$
\tau\left(p_{\xi_{0}}(f(z))\right)-\tau\left(p_{\xi_{0}}(z)\right)>0 .
$$

Choosing $\delta>0$ such that

$$
z \in[0,1]^{2} \Rightarrow \tau\left(p_{\xi_{0}}(f(z))\right)-\tau\left(p_{\xi_{0}}(z)\right) \geq \delta,
$$

one can find a neighborhood $\Xi^{\prime} \subset \Xi$ (which can be chosen convex) of $\xi_{0}$ for the $C^{0}$-topology such that for every smooth vector field $\xi \in \Xi^{\prime}$,

$$
z \in[0,1]^{2} \Rightarrow \tau\left(p_{\xi}(f(z))\right)-\tau\left(p_{\xi}(z)\right) \geq \delta / 2 .
$$

Using the relations

$$
p_{\xi} \circ T=T \circ p_{\xi}, \quad p_{\xi} \circ T^{\prime}=g_{\xi}^{-1} \circ p_{\xi},
$$

one deduces that

$$
\tau\left(p_{\xi}(f(z))\right)-\tau\left(p_{\xi}(z)\right)>0
$$

for every $z \in \mathbb{R}^{2}$.

Lemma 5.5. There exists a smooth vector field $\xi_{1} \in \Xi^{\prime}$ with $\varrho_{\xi_{1}}=\varrho_{\xi_{0}}$.

Proof. Consider two smooth vector fields $\xi_{+}$and $\xi_{-}$in $\Xi^{\prime}$ such that for every $z \in \mathbb{R}^{2}$,

$$
\xi_{0}(z) \wedge \xi_{-}(z)<0<\xi_{0}(z) \wedge \xi_{+}(z) .
$$

One deduces that for any $z \in \Gamma_{0}$,

$$
\tau\left(g_{\xi_{-}}(z)\right)<\tau\left(g_{\xi_{0}}(z)\right)<\tau\left(g_{\xi_{+}}(z)\right),
$$

which implies that $\varrho_{\xi_{-}}<\varrho_{\xi_{0}}<\varrho_{\xi_{+}}$because $\varrho_{\xi_{0}} \notin \mathbb{Q}$. Therefore we can find in the family $\left(s \xi_{-}+(1-s) \xi_{+}\right)_{s \in[0,1]}$ a smooth vector field $\xi_{1}$ such that $\varrho_{\xi_{1}}=\varrho_{\xi_{0}}$.

Lemma 5.6. There exists a smooth vector field $\xi_{2} \in \Xi^{\prime}$ with $\varrho_{\xi_{2}}=\varrho_{\xi_{0}}$ whose foliation is smoothly conjugate to a linear one.

Proof. The induced vector field $\widetilde{\xi}_{1}$ on $\mathbb{T}^{2}$ being $C^{\infty}$, the first return map on $\widetilde{\Gamma}$ is conjugate to a rotation: there exists a homeomorphism $h_{1}: \Gamma_{0} \rightarrow \mathbb{R}$ such that

$$
h_{1}(T(z))=h_{1}(z)+1, \quad h_{1}\left(g_{\xi_{1}}(z)\right)=h_{1}(z)+\varrho_{\xi_{0}} .
$$

One can approximate $h_{1}$ by a smooth diffeomorphism $h_{2}: \Gamma_{0} \rightarrow \mathbb{R}$ satisfying $h_{2}(T(z))=h_{2}(z)+1$ for every $z \in \Gamma_{0}$. Defining $\theta_{\varrho_{\xi_{0}}}: s \mapsto s+\varrho_{\xi_{0}}$, one sees that $h_{2}^{-1} \circ \theta_{\varrho_{\xi_{0}}} \circ h_{2}$ is $C^{0}$-close to $g_{\xi_{1}}$. Using the existence of a tubular 
neighborhood $W$ of $\widetilde{\Gamma}$ and of a smooth diffeomorphism sending $W$ onto $\mathbb{T}^{1} \times[-1,+1]$, the circle $\widetilde{\Gamma}$ onto $\mathbb{T}^{1} \times\{0\}$, and the vector field $\left.\widetilde{\xi}_{1}\right|_{W}$ to a constant vertical vector field, one constructs a smooth vector field $\xi_{2} \in \Xi^{\prime}$ such that

$$
g_{\xi_{2}}=h_{2}^{-1} \circ \theta_{\varrho_{\xi_{0}}} \circ h_{2},
$$

in the same way that we foliated the bricks $\beta_{e}$ in the proof of Lemma 5.2.

Proof of Theorem 0.1. The map

$$
H_{2}=h_{2} \circ p_{\xi_{2}}
$$

is a smooth map satisfying

$$
H_{2}(T(z))=H_{2}(z)+1, \quad H_{2}\left(T^{\prime}(z)\right)=H_{2}(z)-\varrho_{\xi_{0}}
$$

and

$$
H_{2}(f(z))>H_{2}(z)
$$

for every $z \in \mathbb{R}^{2}$. Moreover the form $d H_{2}$ does not vanish and defines on $\mathbb{T}^{2}$ a closed form of cohomology class $\kappa$.

\section{References}

[BCLP] F. Béguin, S. Crovisier, F. Le Roux and A. Patou, Pseudo-rotations of the closed annulus: variation on a theorem of J. Kwapisz, Nonlinearity 17 (2004), 14271453.

[Bo] M. Bonino, A Brouwer-like theorem for orientation reversing homeomorphisms of the sphere, Fund. Math. 182 (2004), 1-40.

[Br] L. E. J. Brouwer, Beweis des ebenen Translationssatzes, Math. Ann. 72 (1912), $37-54$.

[D] E. Doeff, Rotation measures for homeomorphisms of the torus homotopic to a Dehn twist, Ergodic Theory Dynam. Systems 17 (1997), 575-591.

[DM] E. Doeff and M. Misiurewicz, Shear rotation numbers, Nonlinearity 10 (1997), $1755-1762$.

[Fl] M. Flucher, Fixed points of measure preserving torus homeomorphisms, Manuscripta Math. 68 (1990), 271-293.

[Fra] J. Franks, A new proof of the Brouwer plane translation theorem, Ergodic Theory Dynam. Systems 12 (1992), 217-226.

[Fri] D. Fried, The geometry of cross sections to flows, Topology 21 (1982), 353-371.

$[\mathrm{J}] \quad$ B. Jiang, Lectures on Nielsen Fixed Point Theory, Contemp. Math. 14, Amer. Math. Soc., 1983.

[Ke] B. Kerékjártó, Vorlesungen über Topologie (I), Springer, Berlin, 1923.

[Kw] J. Kwapisz, A priori degeneracy of one-dimensional rotation sets for periodic point free torus maps, Trans. Amer. Math. Soc. 354 (2002), 2865-2895.

[LeC1] P. Le Calvez, Propriétés dynamiques des difféomorphismes de l'anneau et du tore, Astérisque 204 (1991).

[LeC2] - Une version feuilletée du théorème de translation de Brouwer, Comment. Math. Helv. 21 (2004), 229-259. 
[LeC3] P. Le Calvez, Une version feuilletée équivariante du théorème de translation de Brouwer, preprint.

[LS] P. Le Calvez et A. Sauzet, Une démonstration dynamique du théorème de translation de Brouwer, Expo. Math. 14 (1996), 277-287.

[LY] P. Le Calvez et J.-C. Yoccoz, Un théorème d'indice pour les homéomorphismes au voisinage d'un point fixe, Ann. of Math. 146 (1997), 241-293.

[LeR] F. Le Roux, Homéomorphismes de surfaces, théorème de la fleur de Leau-Fatou et de la variété stable, Astérisque 292 (2004).

[MZ] M. Misiurewicz and K. Ziemian, Rotation sets for maps of tori, J. London Math. Soc. 40 (1989), 490-506.

[Sa] A. Sauzet, Application des décompositions libres à l'étude des homéomorphismes de surface, thèse de l'Univ. Paris 13, 2001.

[Sc] S. Schwartzman, Asymptotic cycles, Ann. of Math. 68 (1957), 270-284.

Laboratoire Analyse, Géométrie et Applications

C.N.R.S.-U.M.R 7539

Institut Galilée

Université Paris 13

Avenue J.-B. Clément

93430 Villetaneuse, France

E-mail: lecalvez@math.univ-paris13.fr

Received 7 January 2005;

in revised form 19 September 2005 